\title{
Very extended cold gas, star formation and outflows in the halo of a bright quasar at $z>6^{\star}$
}

\author{
C. Cicone $e^{1,2}$, R. Maiolino ${ }^{1,2}$, S. Gallerani ${ }^{3}$, R. Neri ${ }^{4}$, A. Ferrara ${ }^{3}$, E. Sturm ${ }^{5}$, F. Fiore ${ }^{6}$, E. Piconcelli ${ }^{6}$, and C. Feruglio ${ }^{4}$ \\ ${ }^{1}$ Cavendish Laboratory, University of Cambridge, 19 J. J. Thomson Avenue, CB3 OHE Cambridge, UK \\ e-mail: c.cicone@mrao.cam.ac.uk \\ 2 Kavli Institute for Cosmology, University of Cambridge, Madingley Road, CB3 0HA Cambridge, UK \\ 3 Scuola Normale Superiore, Piazza dei Cavalieri 7, 56126 Pisa, Italy \\ 4 Institut de Radioastronomie Millimétrique (IRAM), 300 rue de la Piscine, 38406 St. Martin d'Hères, Grenoble, France \\ 5 Max Planck Institute für Extraterrestrische Physik (MPE), Giessenbachstraße 1, 85748 Garching, Germany \\ ${ }^{6}$ Osservatorio Astronomico di Roma (INAF), via Frascati 33, 00040 Monteporzio Catone, Italy
}

Received 12 September 2014 / Accepted 14 November 2014

\section{ABSTRACT}

\begin{abstract}
Past observations of quasar host galaxies at $z>6$ have found cold gas and star formation on compact scales of a few kiloparsecs. We present new high sensitivity IRAM Plateau de Bure Interferometer follow-up observations of the [C II] $158 \mu \mathrm{m}$ emission line and far-infrared (FIR) continuum in the host galaxy of SDSS J1148+5251, a luminous quasar at redshift 6.4189 . We find that a large portion of the gas traced by [C II] is at high velocities, up to $\sim 1400 \mathrm{~km} \mathrm{~s}^{-1}$ relative to the systemic velocity, confirming the presence of a major outflow as indicated by previous observations. The outflow has a complex morphology and reaches a maximum projected radius of $\simeq 30 \mathrm{kpc}$. The extreme spatial extent of the outflow allows us, for the first time in an external galaxy, to estimate mass-loss rate, kinetic power, and momentum rate of the outflow as a function of the projected distance from the nucleus and the dynamical time scale. These trends reveal multiple outflow events during the past $100 \mathrm{Myr}$, although the bulk of the mass, energy, and momentum appear to have been released more recently within the past 20 Myr. Surprisingly, we discover that the quiescent gas at systemic velocity is also extremely extended. More specifically, we find that, while $30 \%$ of the [C II] within $v \in(-200,200) \mathrm{km} \mathrm{s}^{-1}$ traces a compact component that is not resolved by our observations, $70 \%$ of the $[\mathrm{CII}]$ emission in this velocity range is extended with a projected full width at half maximum (FWHM) size of $17.4 \pm 1.4 \mathrm{kpc}$. We detect FIR continuum emission associated with both the compact and the extended [C II] components, although the extended FIR emission has a FWHM of $11 \pm 3 \mathrm{kpc}$, thus smaller than the extended [C II] source. Overall, our results indicate that the cold gas traced by [C II] is distributed up to $r \sim 30 \mathrm{kpc}$ in the host galaxy of SDSS J1148+5251. A large amount of extended [C II] is likely to be associated with star formation occurring on large scales, but the $[\mathrm{C} \mathrm{II}]$ source extends well beyond the FIR continuum, and additional multi-wavelength observations are needed in order to clarify the origin of this very extended [C II] .
\end{abstract}

Key words. galaxies: general - galaxies: ISM - galaxies: evolution - galaxies: high-redshift - quasars: general

\section{Introduction}

Recent millimetre observations have revealed that the host galaxies of high redshift luminous quasars are quite compact, with extremely intense star formation confined within a few kiloparsecs in their nuclear regions, fed by large amounts of dense gas (e.g. see Carilli \& Walter 2013 for a recent review). Moreover, high resolution interferometric observations of these high redshift sources have suggested the presence of regularly rotating and relatively undisturbed disks (Carniani et al. 2013; Wang et al. 2013), although in some cases they appear to be highly turbulent (De Breuck et al. 2014). However, recent theoretical studies have concluded that, in order to reconcile the extreme properties (black hole ( $\mathrm{BH})$ mass, dust, and gas mass) of these high redshift quasars with early star formation occurring with a standard initial mass function (IMF), the dynamical masses of these objects should be a factor of 3-30 higher than the maximum values allowed by observations (Valiante et al. 2011, 2014; Calura et al. 2014). This suggests that past

* Appendices are available in electronic form at http://www . aanda.org millimetre observations may have missed a significant portion of baryons in high redshift quasar host galaxies.

Only in a few high redshift quasars and radio galaxies have observations revealed the presence of some diffuse gas at a distance of several kiloparsecs from the nucleus, possibly associated with gas-rich companions and suggestive of galaxy merger processes (e.g. Ivison et al. 2008, 2012; Gallerani et al. 2012; Emonts et al. 2014). Within the massive haloes of high-z radio galaxies, cold gas emission can be detected up to tens of kiloparsecs offset from the galactic nucleus, often aligned with the extended radio jets (Nesvadba et al. 2009; Emonts et al. 2011, 2014).

The collisionally excited fine structure transition of $\mathrm{C}^{+}$ ${ }^{2} \mathrm{P}_{3 / 2} \rightarrow{ }^{2} \mathrm{P}_{1 / 2}$ at rest-frame wavelength of $157.741 \mu \mathrm{m}$ (hereafter [C II] $158 \mu \mathrm{m}$ ) is conveniently redshifted at $z>4$ into the submillimetre/millimetre atmospheric windows. The [C II] $158 \mu \mathrm{m}$ is one of the brightest lines in the spectrum of star forming galaxies, since it is the dominant coolant of the interstellar medium (ISM) over a wide range of physical conditions. The ionisation potential of carbon is $11.26 \mathrm{eV}$, so $\mathrm{C}^{+}$, $\mathrm{HI}$, and even $\mathrm{H}_{2}$ usually coexist in the same medium, and indeed neutral hydrogen is the main collisional partner of $\mathrm{C}^{+}$. Although $[\mathrm{C} \mathrm{II}]$ emission 
is commonly associated with star formation (e.g. Sargsyan et al. 2014; De Looze et al. 2014), the large variety of physical conditions in which $\mathrm{C}^{+}$can be produced and excited keeps its interpretation from being straightforward. This has been shown clearly by recent detailed studies of the spatial distribution of various gas tracers in the Milky Way and a few other nearby galaxies, including Herschel observations of [C II]. In particular, in our Galaxy, only $\sim 50 \%$ of the [C II] emission is associated with dense photon-dominated regions (PDRs) exposed to the far-ultraviolet (FUV) radiation field of young and massive stars (Pineda et al. 2013). About 30\% of [C II] in the Milky Way is linked to "CO-dark" molecular hydrogen, which dominates the gas mass of diffuse molecular clouds, but it can also be an important component of some unshielded dense molecular clouds with large envelopes where the formation of $\mathrm{CO}$ is inhibited and where most of the gas-phase carbon is found in $\mathrm{C}^{0}$ or $\mathrm{C}^{+}$atoms (Wolfire et al. 2010; Langer et al. 2010, 2014; Velusamy et al. 2010; Pineda et al. 2013). Finally, Pineda et al. (2013) have shown that $20 \%$ of [C II] is linked to diffuse atomic clouds (in particular to the cold neutral medium, CNM), and a low fraction $(\sim 4 \%)$ is associated with warm ionised medium (WIM), although the WIM contribution to [C II] emission may be significantly more according to Velusamy \& Langer (2014). Further complicating its interpretation, [C II] can be enhanced by shocks (Appleton et al. 2013). Intriguingly, [C II] also seems to be a good tracer of outflows and, especially, of molecular gas in outflow (Fischer et al. 2010; Contursi et al. 2013; Kreckel et al. 2014).

The first high redshift source in which [C II] emission was ever detected (Maiolino et al. 2005), SDSS J1148+5251 at redshift 6.4189, is one of the most powerful quasars in the Universe, with an intrinsic bolometric luminosity of $L_{\mathrm{AGN}}=5.63 \times 10^{13} L_{\odot}$ (Schneider et al. 2014). This is the perfect laboratory for studying the formation of the first massive galaxies and the coevolution of galaxies and black holes at the end of the reionisation epoch. SDSS J1148+5251 was discovered through SDSS photometry (Fan et al. 2003), and since then it has been the subject of extensive multi-wavelength studies, aimed at constraining the extreme properties of its host galaxy and of its cosmological environment (e.g. Bertoldi et al. 2003; Walter et al. 2003, 2004; Willott et al. 2003; Riechers et al. 2009; Gallerani et al. 2010; Decarli et al. 2012; Carilli \& Walter 2013). Walter et al. (2009) observed the [C II] emission line in SDSS J1148+5251, using the most extended configuration of the IRAM Plateau de Bure Interferometer (PdBI), and derived a size of $1.5 \mathrm{kpc}$, which combined with the extreme far-infrared (FIR) luminosity of this source, suggests the presence of a kpc-scale "hyperstarburst" in its nucleus, fed by large amounts of molecular gas. Moreover, SDSS J1148+5251 was also the first high redshift galaxy in which a powerful quasar-driven outflow was discovered (Maiolino et al. 2012). The outflow was revealed by the presence of extremely broad wings of the [C II] emission line, tracing gas at high velocities ( $v$ up to $1200 \mathrm{~km} \mathrm{~s}^{-1}$ ), which appeared marginally resolved at a 2 arcsec resolution, with a measured (projected) diameter of $\sim 16 \mathrm{kpc}$.

In this work we present new follow-up observations of the [C II] emission line and FIR continuum in SDSS J1148+5251, obtained with the $C$ and D configurations of the IRAM PdBI (resulting in a synthesised beam size of $1.3^{\prime \prime} \times 1.2^{\prime \prime}$ for the $[\mathrm{C} \mathrm{II}]$ observations and of $1.1^{\prime \prime} \times 1.0^{\prime \prime}$ for the FIR continuum observations), which enable us to investigate the properties of this source on large scales, with a sensitivity much higher than previous observations. Throughout the paper, we adopt a standard $\Lambda \mathrm{CDM}$ cosmological model with $H_{0}=67.3 \mathrm{~km} \mathrm{~s}^{-1} \mathrm{Mpc}^{-1}, \Omega_{\Lambda}=0.685$,
$\Omega_{\mathrm{M}}=0.315$ (Planck Collaboration XVI 2014). With the adopted cosmology, at the redshift of SDSS J1148+5251, the physical scale is $5.623 \mathrm{kpc} \operatorname{arcsec}^{-1}$, and the age of the Universe is 0.85 Gyr.

\section{Observations}

We have observed with the IRAM PdBI the [C II] $158 \mu \mathrm{m}$ line in the host galaxy of the luminous quasar SDSS J1148+5251. The pointing and phase centre of our observations corresponds to the SDSS optical position of the quasar ( $\mathrm{RA}=11: 48: 16.64$, $\operatorname{Dec}=52: 51: 50.30)$. At the redshift of the source $(z=6.4189$, Maiolino et al. 2005), the [C II] $158 \mu \mathrm{m}$ transition is redshifted into the $1 \mathrm{~mm}$ band, at an observed frequency of $256.175 \mathrm{GHz}$. The observations were obtained with the $C$ configuration of the IRAM PdBI (with 6 antennas covering baselines from $16.6 \mathrm{~m}$ to $176.0 \mathrm{~m}$ ) between November 2012 and April 2013 and were combined with the previous 2011 observations in D configuration (Maiolino et al. 2012), for a total on-source-time of $30 \mathrm{~h}$. We reach a sensitivity of $0.46 \mathrm{mJy} \mathrm{beam}^{-1}$ in channels of $100 \mathrm{~km} \mathrm{~s}^{-1}$, which is unprecedented for IRAM PdBI observations of high redshift quasars at this frequency (Wang et al. 2013 reach a similar line sensitivity using the Atacama large millimetre/sub-millimetre array (ALMA)). The resulting synthesised beam-size using natural weighting is $1.3^{\prime \prime} \times 1.2^{\prime \prime}$, and the PdBI primary beam, giving the field of view, at these frequencies is $19.7^{\prime \prime} \times 19.7^{\prime \prime}$. Data reduction and analysis were performed by using the CLIC and MAPPING softwares within the GILDAS package. The flux calibration accuracy in the $1 \mathrm{~mm}$ band is about 20\% (Castro-Carrizo \& Neri 2010). We used the wide-band express (WideX) correlator offered by the PdBI, which provides a spectral resolution of $20 \mathrm{MHz}$ (corresponding to $23.4 \mathrm{~km} \mathrm{~s}^{-1}$ ) over its full bandwidth of $3.6 \mathrm{GHz}$. The $3.6 \mathrm{GHz}$ spectral window was centred at $256.172 \mathrm{GHz}$ (i.e. on the [C II] $158 \mu \mathrm{m}$ line) for all tracks but two, for which the centre was instead set at $256.672 \mathrm{GHz}$, hence providing an additional $0.5 \mathrm{GHz}$-wide window on the continuum blueward of the line.

In this work we also make use of ancillary $\mathrm{C}+\mathrm{D}$ configuration IRAM PdBI observations of the FIR continuum of SDSS J1148+5251 at $262 \mathrm{GHz}$ (rest frame wavelength $\lambda=154.2 \mu \mathrm{m}$ ) and $259.4 \mathrm{GHz}$ (rest frame wavelength $\lambda=$ $155.8 \mu \mathrm{m}$ ), carried out between May 2010 and December 2012. By merging these two datasets at $262 \mathrm{GHz}$ and $259.4 \mathrm{GHz}$, we obtain a high sensitivity ( $1 \sigma \mathrm{rms}$ noise of $0.082 \mathrm{mJy}^{\mathrm{beam}}{ }^{-1}$ ) map of the FIR continuum of SDSS J1148+5251 at an average rest-frame wavelength $\lambda=155 \mu \mathrm{m}$ (average observed frequency of $261 \mathrm{GHz}$ ). The resulting synthesised beam-size using natural weighting is $1.1^{\prime \prime} \times 1.0^{\prime \prime}$. The total on source time for these ancillary FIR observations is $28 \mathrm{~h}$. These deep FIR continuum observations are part of a parallel IRAM PdBI programme. The dataset at $262 \mathrm{GHz}$ has been presented by Gallerani et al. (2014), who report the serendipitous detection of the $\mathrm{CO}(17-16)$ and $\mathrm{OH}^{+}$emission lines in SDSS J1148+5251.

\section{Results}

\subsection{The FIR continuum at $256 \mathrm{GHz}$}

For the purpose of continuum subtraction, the rest-frame $157.7 \mu \mathrm{m}$ FIR continuum (observed frequency of $256 \mathrm{GHz}$ ) is estimated by collapsing the line-free channels of our wide-band IRAM PdBI observations, corresponding to the velocity ranges of $v \in(-2700,-2400) \mathrm{km} \mathrm{s}^{-1}, v \in(-1900,-1500) \mathrm{km} \mathrm{s}^{-1}$ and $v \in(1500,2100) \mathrm{km} \mathrm{s}^{-1}$. The channels between 

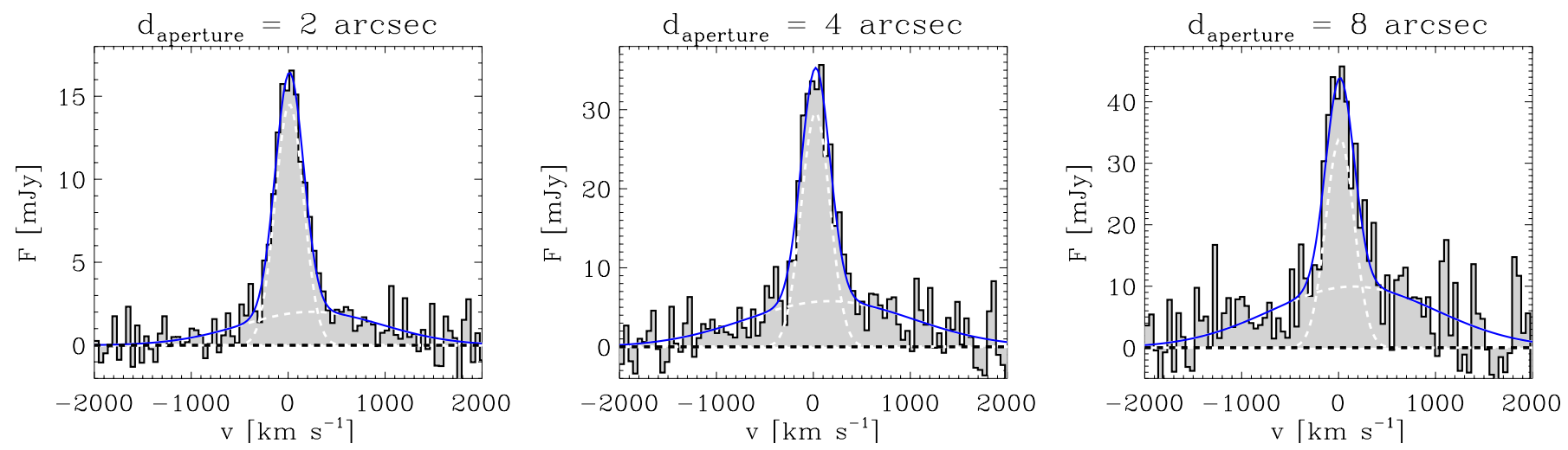

Fig. 1. IRAM PdBI continuum-subtracted spectrum of the [C II] $158 \mu \mathrm{m}$ emission line of SDSS J1148+5251. The three spectra shown have been extracted using different apertures, namely circular apertures with diameters of 2 arcsec (left panel), 4 arcsec (central panel) and 8 arcsec (right panel). Note the different scales on the $y$-axes in the three panels. For display purposes, the spectra have been re-binned by a factor of 2 , corresponding to channels of $46.8 \mathrm{~km} \mathrm{~s}^{-1}$. The Gaussian fits to the line profiles (using a narrow and a broad Gaussian component to fit respectively the narrow core, tracing quiescent gas, and the broad wings, tracing the outflow) are performed on the original non-binned spectra and the fits results are reported in Table 1 .

Table 1. Results of the Gaussian fits to the [C II] $158 \mu \mathrm{m}$ emission line spectrum of SDSS J1148+5251.

\begin{tabular}{|c|c|c|c|c|c|c|c|c|c|}
\hline \multirow[t]{2}{*}{ Aperture $^{\dagger}$} & \multicolumn{4}{|c|}{ Narrow component } & \multicolumn{4}{|c|}{ Broad component } & \multirow{2}{*}{$\begin{array}{c}\text { Total line } \\
I_{v} \\
{\left[\mathrm{Jy} \mathrm{km} \mathrm{s}^{-1}\right]}\end{array}$} \\
\hline & $\begin{array}{c}v \\
{\left[\mathrm{~km} \mathrm{~s}^{-1}\right]}\end{array}$ & $\begin{array}{c}\sigma_{v} \\
{\left[\mathrm{~km} \mathrm{~s}^{-1}\right]}\end{array}$ & $\begin{array}{l}S_{\text {peak }} \\
{[\mathrm{mJy}]}\end{array}$ & $\begin{array}{c}I_{v} \\
{\left[\mathrm{Jy} \mathrm{km} \mathrm{s}^{-1}\right]}\end{array}$ & $\begin{array}{c}v \\
{\left[\mathrm{~km} \mathrm{~s}^{-1}\right]}\end{array}$ & $\begin{array}{c}\sigma_{v} \\
{\left[\mathrm{~km} \mathrm{~s}^{-1}\right]}\end{array}$ & $\begin{array}{c}S_{\text {peak }} \\
{[\mathrm{mJy}]}\end{array}$ & $\begin{array}{c}I_{v} \\
{\left[\mathrm{Jy} \mathrm{km} \mathrm{s}^{-1}\right]}\end{array}$ & \\
\hline $2 \operatorname{arcsec}$ & $13 \pm 9$ & $146 \pm 11$ & $14.5 \pm 0.9$ & $5.3 \pm 0.5$ & $250 \pm 160$ & $730 \pm 170$ & $2.0 \pm 0.6$ & $3.7 \pm 1.4$ & $9.0 \pm 1.5$ \\
\hline $4 a$ & 24 & \pm 16 & 30 & 11.0 & 17 & $900 \pm$ & $5.8=$ & & \\
\hline $8 \operatorname{arcsec}$ & $14 \pm 19$ & $150 \pm 20$ & $34 \pm 4$ & $13 \pm 3$ & $200 \pm 300$ & $900 \pm 300$ & $10 \pm 3$ & $21 \pm 9$ & $34 \pm 9$ \\
\hline
\end{tabular}

Notes. ${ }^{(\dagger)}$ Diameter of the circular aperture used for extracting the spectrum, as in Fig. 1.

$v=-2400 \mathrm{~km} \mathrm{~s}^{-1}$ and $v=-1900 \mathrm{~km} \mathrm{~s}^{-1}$ are excluded from the continuum integration because of the presence of a putative feature, detected at the $4.2 \sigma$ level in the maps, whose position is shifted by 1.5 arcsec to the south with respect to the quasar optical position and whose origin is not clear from our data (it could be be either part of the extended outflow discussed in Sect. 3.4 or associated with another galaxy).

The $u v$ plot of the continuum at $256 \mathrm{GHz}$ can be fitted by a point (unresolved) source $^{1}$ centred at $\mathrm{RA}=11: 48: 16.62$ and $\operatorname{Dec}=52: 51: 50.22$, with a flux density of $3.3 \mathrm{mJy}$. We compare our new continuum flux density estimate with the Max Planck millimetre bolometer-II (MAMBO-II) $1.2 \mathrm{~mm}$ observations of Bertoldi et al. (2003), who report a flux density of 5.0 $\pm 0.6 \mathrm{mJy}$. Because of the broad bandwidth of MAMBO-II, the flux expected at our PdBI frequency of $256 \mathrm{GHz}$ from the MAMBO-II observations of Bertoldi et al. (2003) must be modelled by taking into account both the MAMBO-II transmission curve, which determines the spectral response of the bolometer at different frequencies, and the FIR spectral energy distribution (SED) of SDSS J1148+5251, which, at FIR wavelengths, can be modelled by using a modified blackbody curve with emissivity $\beta=1.6$ (Leipski et al. 2014). For simplicity, we perform the calculation under the Rayleigh-Jeans approximation and by assuming $\tau_{v} \ll 1$ so that the flux density scales as $S_{v} \propto v^{(\beta+2)}$, where the optical depth is parametrised as a power law in frequency,

\footnotetext{
1 We note that, although the FIR continuum of SDSS J1148+5251 is not resolved in the $256 \mathrm{GHz}$ dataset, the FIR continuum emission appears well resolved in the deeper ancillary dataset at $261 \mathrm{GHz}$ $\left(\lambda_{\text {rest }}=155 \mu \mathrm{m}\right.$, see also explanation in Sect. 2), as shown in Sect. 3.6.
}

$\tau_{v}=\left(v / v_{0}\right)^{\beta}$, following Draine $(2006)^{2}$. We obtain an expected flux density at $256 \mathrm{GHz}$ of $4.8 \pm 0.6 \mathrm{mJy}$, to be compared with our measured value of $3.3 \pm 0.7 \mathrm{mJy}$, where the error includes a $20 \%$ flux calibration uncertainty. We note that there is a small inconsistency between our new $157.7 \mu \mathrm{m}$ continuum estimate and the flux density expected at these wavelengths from the bolometric observations of Bertoldi et al. (2003). This discrepancy was not evidenced in Maiolino et al. (2012) because of the larger error of their continuum measure, which was only based on the D-configuration data. This small inconsistency with Bertoldi et al. (2003) is likely due to the presence of additional sources close to SDSS J1148+5251 contributing to the 10.7" MAMBO-II beam. In particular, one source $\sim 10^{\prime \prime}$ north-west of SDSS J1148+5251 was clearly detected by Herschel (Leipski et al. 2010, 2013), and it is also marginally detected by our PdBI observations (Fig. A.1). In Appendix A we further test this hypothesis and we show that, when modelling the FIR SED of SDSS J1148+5251 by only considering the Herschel/PACS continuum measurements of Leipski et al. (2013), corrected for the contaminating north-western source, and the PdBI measurements by Riechers et al. (2009) and Gallerani et al. (2014) at other frequencies, the flux density expected at the MAMBO-II frequencies (by taking into account the full transmission curve) would be of $3.8 \mathrm{mJy}$, which in turn would predict a flux density at $256 \mathrm{GHz}$ of $3.6 \pm 0.4 \mathrm{mJy}$, consistent with our PdBI measurement.

\footnotetext{
$2 \lambda_{0}=c / v_{0}$ is the wavelength where the optical depth is unity, expected to be $\lambda_{0} \simeq 100 \mu \mathrm{m}$.
} 


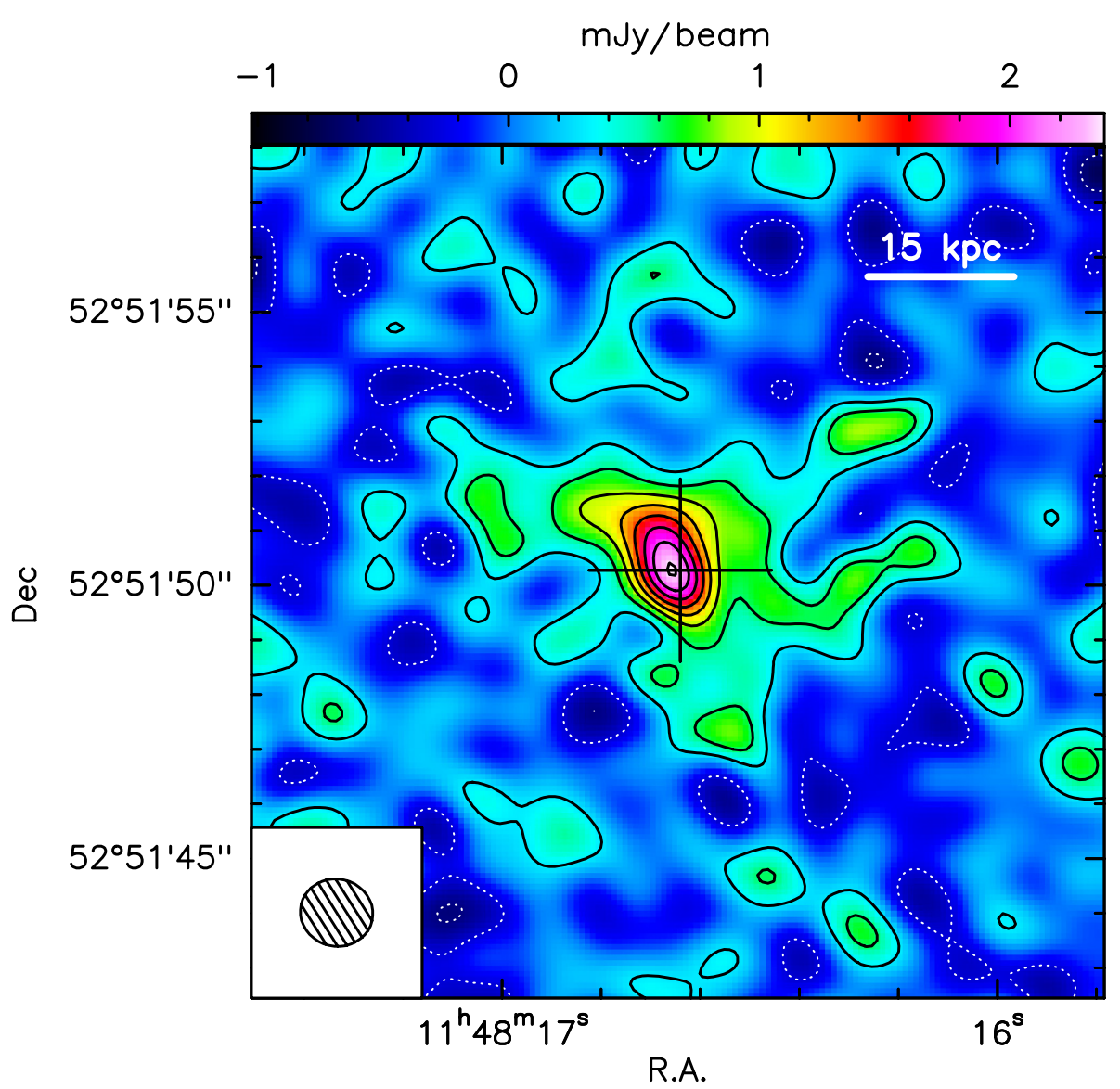

Fig. 2. IRAM PdBI continuum-subtracted map of the total [C II] $158 \mu \mathrm{m}$ emission of SDSS J1148+5251, integrated within $v \in(-1400,1200)$ $\mathrm{km} \mathrm{s}^{-1}$. Negative and positive contours are in steps of $3 \sigma\left(1 \sigma \mathrm{rms}\right.$ noise is $0.26 \mathrm{Jy}$ beam $\left.{ }^{-1} \mathrm{~km} \mathrm{~s}^{-1}\right)$. The synthesised beam $\left(1.3^{\prime \prime} \times 1.2^{\prime \prime}\right)$ is shown in the bottom left corner of the map. The cross indicates the pointing and phase centre, corresponding to the optical position of the quasar.

\subsection{The total [C II] $158 \mu \mathrm{m}$ emission}

Following the standard procedure, we subtract the continuum (estimated in Sect. 3.1) directly from the $u v$ data, and we extract from the continuum-subtracted and cleaned data cube the spectrum of the $[\mathrm{CII}] 158 \mu \mathrm{m}$ emission line of SDSS J1148+5251. In Fig. 1 we show the resulting continuumsubtracted spectra extracted using three circular apertures with diameters of 2 arcsec, 4 arcsec, and 8 arcsec. Note that the three plots in Fig. 1 have different scales on the $y$-axis. Each spectrum in Fig. 1 is fit by using two Gaussians, e.g., a narrow Gaussian to fit the central core of the line, tracing quiescent gas, and a broad Gaussian to fit the broad wings, tracing the outflow discovered by Maiolino et al. (2012). The fit with two Gaussians is a simplistic model for the [C II] $158 \mu \mathrm{m}$ emission profile of SDSS J1148+5251, however it constitutes a good starting point for our study. The fit results are reported in Table 1 . The widths of the two Gaussians used for fitting the [C II] $158 \mu \mathrm{m}$ spectral profile show no significant variations between spectra extracted from different apertures ${ }^{3}$, and they are consistent with Maiolino et al. (2012).

Figure 1 and Table 1 clearly show that the contribution from an extended gas component to the total [C II] $158 \mu \mathrm{m}$ flux in SDSS J1148+5251 is significant. Indeed, the total integrated flux estimated by using the spectrum extracted from the 8 arcsec

\footnotetext{
3 We note that all the six parameters of the fit are left as free parameters in the spectral fitting procedure. In particular, we did not constrain the fits performed on the spectra extracted from different apertures to have same widths, central velocities and/or amplitudes.
}

diameter aperture is significantly larger than the flux retrieved using the 4 arcsec aperture, and is almost four times as large as the flux measured from the 2 arcsec aperture. Such flux increment is observed in both the narrow and the broad Gaussians that are fit to the emission line profiles, suggesting that both components trace emission extended on extremely large scales ( $d \gtrsim 4$ arcsec on sky, corresponding to $d \gtrsim 22 \mathrm{kpc}$ ). However, we note that the largest flux variation with aperture size is observed in the broad wings of the [C II] $158 \mu \mathrm{m}$ line, confirming that the massive outflow is very extended.

The complex morphology and extreme spatial extent of the [C II] $158 \mu \mathrm{m}$ emission around this $z \sim 6.4$ quasar are clearly revealed by the map in Fig. 2, obtained by collapsing the velocity channels spanning the full velocity extent of the emission line profile, i.e. from $v=-1400 \mathrm{~km} \mathrm{~s}^{-1}$ to $v=1200 \mathrm{~km} \mathrm{~s}^{-1}$. The map shows a filamentary [C II] $158 \mu \mathrm{m}$ source, with structures extending in the radial direction up to the extraordinary (for a quasar host galaxy at $z=6.4$ ) (projected) distances of $r \sim 30 \mathrm{kpc}$ from the quasar position (indicated by the cross).

The fits performed on the spectra extracted from different apertures (Fig. 1 and Table 1) suggest that a large amount of the [C II $158 \mu \mathrm{m}$ emission at a distance $r>2$ arcsec from the quasar (i.e. the emission that is not retrieved by using the 4 arcsec diameter aperture, but that is recovered with the largest 8 arcsec diameter aperture) is associated with the broad wings of the [C II] line, tracing a powerful outflow (Maiolino et al. 2012). We further investigate this in Fig. 3, where we show the same map as Fig. 2, in which we have marked the position of six regions (indicated with $\mathrm{A}-\mathrm{F}$ ) that are at a distance of $r \gtrsim 2 \operatorname{arcsec}$ 

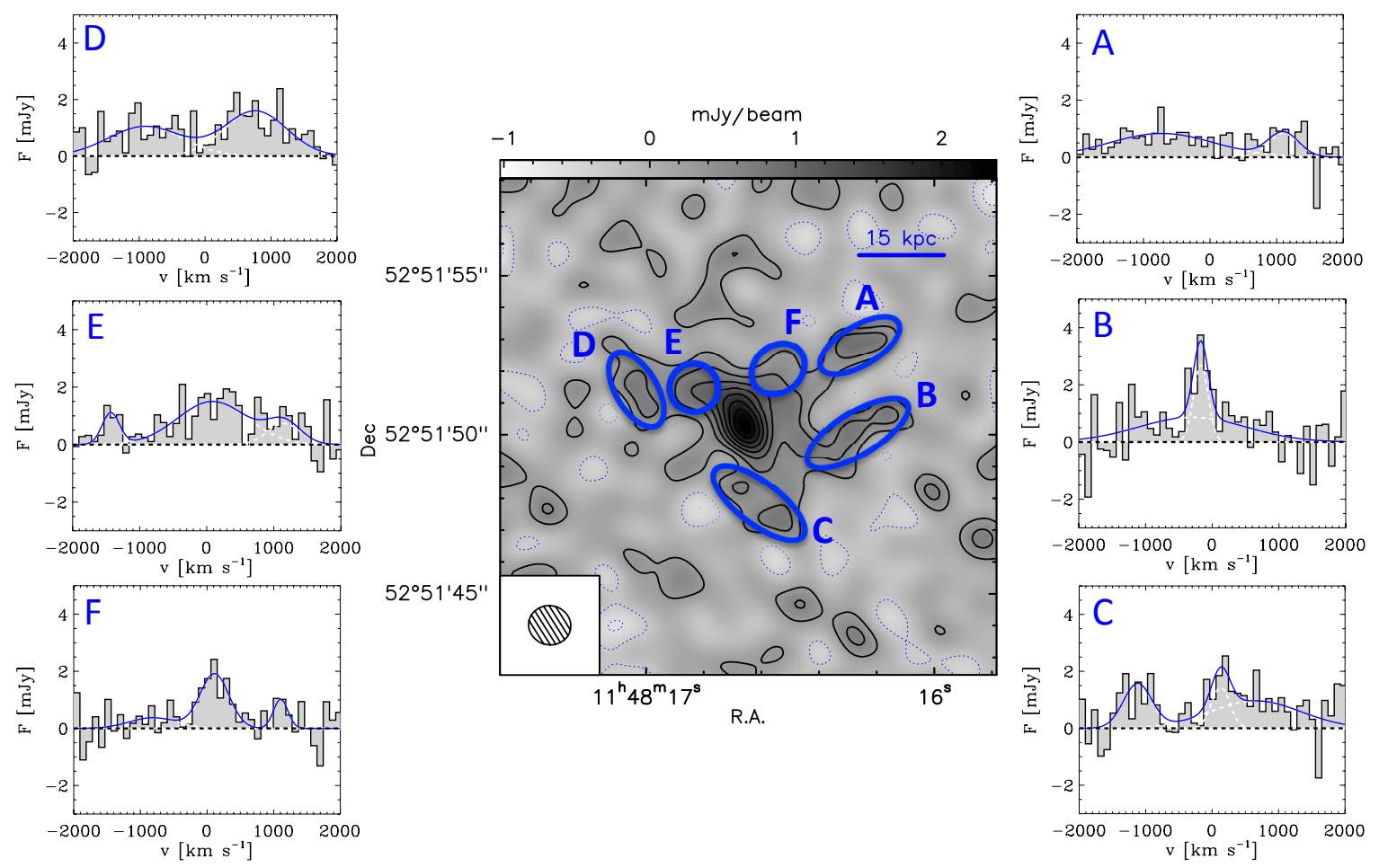

Fig. 3. IRAM PdBI continuum-subtracted map of the total [C II] $158 \mu \mathrm{m}$ emission of SDSS J1148+5251, integrated within $v \in$ $(-1400,1200) \mathrm{km} \mathrm{s}^{-1}$, along with spectra extracted from different positions on the source. Negative and positive contours are in steps of $3 \sigma$ ( $1 \sigma \mathrm{rms}$ noise is $\left.0.26 \mathrm{Jy} \mathrm{beam}^{-1} \mathrm{~km} \mathrm{~s}^{-1}\right)$. For display purposes, the spectra have been re-binned by a factor of 4 , corresponding to channels of $93.6 \mathrm{~km} \mathrm{~s}^{-1}$. The Gaussian fits to the line profiles are performed on the original non-binned spectra and the fits results are presented in Table B.1.

from the emission peak. These regions represent the brightest features within the extended [C II] $158 \mu \mathrm{m}$ source. The spectra extracted from regions A-F (by using the elliptical apertures indicated in the map) are also shown in Fig. 3. The spectral fitting, performed by using multiple Gaussians (the fit results are reported in Table B.1 in Appendix B), shows that features A-F are characterised, on average, by high velocity dispersions and velocity offsets, suggesting an outflow origin.

However, there is also a large amount of extended [C II] associated with "narrow" emission at systemic velocity (e.g. features B, C, F in Fig. 3), indicating the presence of extended dynamically "quiescent" (i.e. non outflowing) [C II] -emitting gas outside of the central "hyper-starburst" detected by Walter et al. (2009). This extremely interesting - and unexpected - new prospect is investigated in Sect. 3.5. Interestingly, the narrow $\left(\sigma_{v}=110 \pm 50 \mathrm{~km} \mathrm{~s}^{-1}\right)$ feature in spectrum B with peak flux density of $S_{\text {peak }}=2.7 \pm 1.0 \mathrm{mJy}$ is blueshifted at a velocity of $v=-170 \pm 40 \mathrm{~km} \mathrm{~s}^{-1}$ (Table B.1). The shift in velocity, together with the low velocity dispersion and large distance from the nucleus, suggests that this feature may have a merger/inflow origin. This hypothesis is however difficult to test with the current data.

\subsection{Comparison with previous observations}

We listed in Table 2 all previous and current [C II] observations of SDSS J1148+5251. We note that the total [C II] $158 \mu \mathrm{m}$ velocity-integrated flux of $34 \pm 9 \mathrm{Jy} \mathrm{km} \mathrm{s}^{-1}$ that we estimate from our new IRAM PdBI observations is significantly larger than any previous flux measurement of [C II] obtained for this source. Such large discrepancy deserves further clarification, as these new observations significantly change the scenario presented by previous [C II] $158 \mu \mathrm{m}$ observations of SDSS J1148+5251 and of other high redshift quasars.
Table 2. Comparison with previous [C II] observations of SDSS J1148+5251.

\begin{tabular}{lccc}
\hline \hline Ref. & $\begin{array}{c}\sigma_{v}^{\dagger} \\
{\left[\mathrm{km} \mathrm{s}^{-1}\right]}\end{array}$ & $\begin{array}{c}S_{\text {peak }} \\
{[\mathrm{mJy}]}\end{array}$ & $\begin{array}{c}I_{v} \\
{\left[\mathrm{Jy} \mathrm{km} \mathrm{s}^{-1}\right]}\end{array}$ \\
\hline Maiolino et al. (2005) & $150 \pm 20$ & 12 & $4.1 \pm 0.5$ \\
Walter et al. (2009) & $122 \pm 12$ & $12.7 \pm 1.1$ & $3.9 \pm 0.3$ \\
Maiolino et al. (2012) $)^{\ddagger}$ & $150 \pm 20$ & $23 \pm 2$ & $14 \pm 3$ \\
This work $^{\ddagger}$ & $150 \pm 20$ & $44 \pm 5$ & $34 \pm 9$ \\
\hline
\end{tabular}

Notes. ${ }^{(\dagger)}$ The values of $\sigma_{v}$ reported for Maiolino et al. (2012) and for the present work refer to the narrow component to allow a comparison with earlier studies that did not detect the broad component. ${ }^{*}$ Values obtained from Gaussian fitting of the spectra extracted from the largest apertures used in these works.

The first but only apparent flux inconsistency that we evidence is with the D-configuration data presented in Maiolino et al. (2012). The total flux derived by using the spectrum extracted from a 6 arcsec-diameter aperture shown in Maiolino et al. (2012) is over a factor of two lower than our new estimate (Table 2). This is in part due to the use of a smaller aperture by Maiolino et al. (2012), which likely missed a fraction of the most extended emission. However, we have also reanalysed the Maiolino et al. (2012) dataset (which is also included in the final dataset used in this work), and we have found that the bulk of the flux loss was due to cleaning problems associated with the data cubes, with lower sensitivity, from which the spectra were extracted. Indeed, the discrepancy between the two datasets is much reduced (down to a $\lesssim 20 \%$ flux discrepancy, likely associated with flux calibration issues in the $1 \mathrm{~mm}$ band, e.g. Castro-Carrizo \& Neri 2010) when estimating the fluxes from the $u v$ data. 

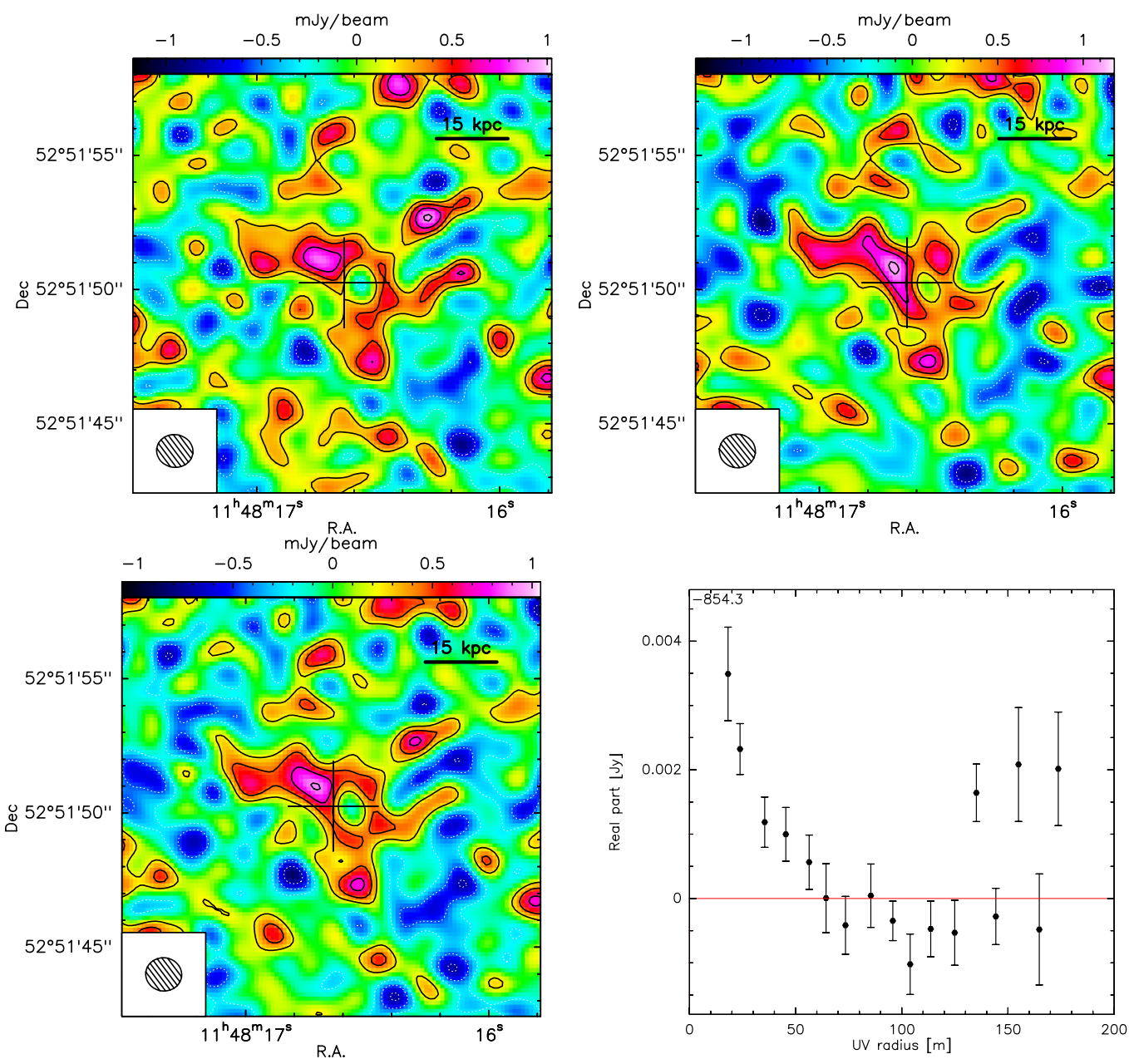

Fig. 4. Top panels: IRAM PdBI continuum-subtracted maps of the blue (left) and red (right) wings of the [C II] $158 \mu \mathrm{m}$ line of SDSS J1148+5251, integrated within $v \in(-1400,-300) \mathrm{km} \mathrm{s}^{-1}$ and $v \in(400,1200) \mathrm{km} \mathrm{s}^{-1}$, respectively. Negative and positive contours are in steps of $1.5 \sigma(1 \sigma \mathrm{rms}$ is $0.17 \mathrm{Jy} \mathrm{beam}^{-1} \mathrm{~km} \mathrm{~s}^{-1}$ and $0.14 \mathrm{Jy} \mathrm{beam}^{-1} \mathrm{~km} \mathrm{~s}^{-1}$ for the blue and red wing, respectively). Bottom panels: map of the blue and red wings merged together (left) and corresponding $u v$ plot (right). In the map of the merged wings, negative and positive contours are in steps of $2 \sigma(1 \sigma$ rms is $0.21 \mathrm{Jy}$ beam ${ }^{-1} \mathrm{~km} \mathrm{~s}^{-1}$ ). The $u v$ plot shows the real part of visibilities as a function of the $u v$ distance, binned in baseline steps of $10 \mathrm{~m}$. In all maps, the cross indicates the pointing and phase centre, corresponding to the optical position of the quasar.

The discrepancy with Walter et al. (2009), who derived a flux of only $3.9 \pm 0.3 \mathrm{Jy} \mathrm{km} \mathrm{s}{ }^{-1}$, can instead be almost entirely explained by the complex and extended nature of the source (Fig. 2). These authors indeed observed SDSS J1148+5251 with the most extended configuration of the IRAM PdBI, therefore lacking short baselines and completely resolving out the extended [C II] $158 \mu \mathrm{m}$ emission. As a result, they only detected the [C II] $158 \mu$ m flux associated with the central "hyperstarburst", which is concentrated within a very compact region of size $\sim 1.5 \mathrm{kpc}$. Moreover, due to their narrow bandwidth of $\Delta v=1 \mathrm{GHz}$, the broad wings associated with the outflow could not be identified by Walter et al. (2009), and were subtracted from the line as continuum emission. This implies that also the flux of the narrow component was underestimated because the continuum level was overestimated, thereby causing an additional significant flux loss with respect to our new observations.

The origin of the discrepancy with the single-dish (IRAM $30 \mathrm{~m}$ ) flux measure by Maiolino et al. (2005) is more uncertain. Similarly to Walter et al. (2009), Maiolino et al. (2005) had a bandwidth of only $1 \mathrm{GHz}$, and so they also subtracted the broad wings from the baselines as continuum emission. However, even taking into account the flux loss due to the wings subtraction, there is still an inconsistency of more than a factor of 2 with respect to our estimate (Table 2). As a consequence, we suspect that the early [C II] $158 \mu$ m detection in SDSS J1148 +5251 by Maiolino et al. (2005) was affected by some calibration issues. In particular, pointing uncertainties may have played an important role. At that time (2004), the lack of bright sources close to SDSS J1148+5251 suitable for pointing calibration, had forced the use of pointing calibration sources very far away from the target, which may have affected significantly the final pointing accuracy. As a consequence, the pointing uncertainty of the IRAM $30 \mathrm{~m}$ observations was probably comparable to or possibly even larger than the nominal upper limit of 3" quoted by the observatory in 2004. Since the full width at half maximum (FWHM) of the IRAM $30 \mathrm{~m}$ beam-size at $256 \mathrm{GHz}$ is only of $9.6^{\prime \prime}$, a pointing offset of $3^{\prime \prime}-4^{\prime \prime}$ could have caused a significant flux loss.

\subsection{The resolved [CII] outflow}

The [C II] $158 \mu \mathrm{m}$ spectrum of SDSS J1148+5251 exhibits extremely broad $\left(F W H M=2100 \pm 500 \mathrm{~km} \mathrm{~s}^{-1}\right)$ wings, superimposed on a narrower core with $F W H M=350 \pm 50 \mathrm{~km} \mathrm{~s}^{-1}$ (Fig. 1). The broad wings of the [C II] $158 \mu \mathrm{m}$ line, discovered by Maiolino et al. (2012), are believed to trace the most distant and powerful quasar-driven outflow ever detected. The outflow 
is also extremely extended. This could already be inferred from the low spatial resolution (synthesised beam of $2.2^{\prime \prime} \times 1.8^{\prime \prime}$ ) observations presented in Maiolino et al. (2012), which marginally resolved the emission associated with the broad wings, providing a rough estimate for the outflow size (FWHM) of 2.9 arcsec.

Our new, higher resolution IRAM PdBI observations fully resolve the [C II] $158 \mu$ m outflow of SDSS J1148+5251. In the top panels of Fig. 4 we show the continuum-subtracted maps of the blue and red wings of the [C II] line, integrated within $v \in(-1400,-300) \mathrm{km} \mathrm{s}^{-1}$ and $v \in(400,1200) \mathrm{km} \mathrm{s}^{-1}$, respectively. We also report in the bottom-left panel of Fig. 4 the map of the wings merged together, similarly to Maiolino et al. (2012). As already suggested by the analysis of the spectra extracted from different apertures (Fig. 1 and Table 1), the maps unambiguously show that the wings are extremely extended, and in particular that they are even more extended than what initially estimated by Maiolino et al. (2012). The bottom-right panel of Fig. 4 shows the real part of the visibilities of the broad wings as a function of the $u v$ baseline radius (also known as " $u v$ plot"). The steeply declining trend clearly confirms that the broad wings are extended and resolved by our observations at high significance, and that they are not an artefact of the cleaning process. We note that the detection of the broad and spatially extended [C II] component, tracing the outflow, is also confirmed by splitting the dataset into independent parts.

The fluctuations observed at large $u v$ radii in the $u v$ plot of the broad wings (bottom-right panel of Fig. 4) indicate that the morphology of the outflow is complex, non-Gaussian, consistently with the complexity of the structures revealed by the maps. For example, both a disk-like and a ring-like brightness distributions would produce similar positive and negative fluctuations as a function of $u v$ radius, although we found that neither of these models provides a good fit to our data.

The maps of the blue and red wings of the [C II] $158 \mu \mathrm{m}$ emission (upper panels of Fig. 4) indicate that the blueshifted and the redshifted sides of the outflow are generally co-spatial. Indeed, the two maps trace the same butterfly-shaped structure, although some blobs appear brighter in the blue component than in the red one and viceversa. We note that the spatial correspondence between blueshifted and redshifted wings rules out the hypothesis that companion galaxies with different systemic velocities are at the origin of the bulk of the broad [C II] $158 \mu \mathrm{m}$ emission, leaving a massive and extended outflow as the only viable explanation.

\subsubsection{Outflow velocity and dynamical time scale}

In Maiolino et al. (2012) the outflow was only marginally resolved, and the outflow mass-loss rate of $\dot{M}_{\text {out }} \gtrsim 3500 M_{\odot} \mathrm{yr}^{-1}$ was estimated by assuming a spherical and/or multi-conical geometry. In the model adopted by Maiolino et al. (2012), the outflow rate was given by $\dot{M}_{\text {out }}=3 v M_{\text {out }} / R$, where $v$ is the outflow velocity, $M_{\text {out }}$ is the mass of atomic gas in outflow (estimated from the [C II] $158 \mu \mathrm{m}$ flux in the broad wings), and $R$ is the radius of the broad wings emission (obtained by fitting the $u v$ plot of the broad wings with a circular Gaussian model). However, our new, higher resolution IRAM PdBI observations allow us to fully resolve the outflow, hence enabling a much more detailed characterisation of the outflow properties, which was not possible with the previous dataset. The complex morphology revealed by the new maps of the outflow (Fig. 4) is clearly not consistent with the simple spherical or multi-conical geometry assumed by Maiolino et al. (2012). Any attempt of modelling the [C II] outflow in SDSS J1148+5251 based on the current data would require a different, more complex geometry, and the uncertainties on such model would be very large. For this reason, in this paper, we decide to abstain from a simplistic modelling of the outflow geometry and instead we adopt a different, "resolved" approach for estimating the outflow properties.

Our approach is described in detail in Appendix C, and is based on measuring the dynamical time scales of individual "blobs" of [C II] emission within the outflow ${ }^{4}$. More specifically, we produce channel maps centred at different velocities, spanning the entire velocity range corresponding to the red and blue wings of the [C II] $158 \mu \mathrm{m}$ line, tracing the outflow in the host galaxy of SDSS J1148+5251. The outflow channel maps are shown in Fig. C.1. In these maps, we identify 48 "clumps" belonging to the outflow, as explained in Appendix C. For each clump in outflow we estimate its average (projected) velocity $(v)$, its (projected) distance from the quasar $(R)$, and its [C II] flux, which is used for estimating the mass of (atomic) gas in outflow as explained in the next section (Sect. 3.4.2). The dynamical time scale of a clump in outflow is therefore simply given by $\tau_{\text {dyn }}=R / v$. We note that, by applying this definition, we are implicitly assuming that the velocity $v$ of the clumps is constant throughout the outflow.

The relationship between the velocity and the distance from the quasar of the clumps in outflow is shown in Fig. 5. The average velocity of the outflowing blobs does not depend on $R$, suggesting that the outflow is not significantly accelerating and/or decelerating. This also supports the definition of dynamical time scales given above. The distribution of $\tau_{\text {dyn }}$ within the outflow is reported in Fig. 6; the histogram shows a slightly skewed left distribution, with median of $\tau_{\text {dyn }}^{\text {median }}=10^{7.4} \mathrm{yr}$, but spanning a broad range from $\tau_{\text {dyn }}^{\min }=10^{6.6} \mathrm{yr}$ to $\tau_{\text {dyn }}^{\max }=10^{8.0} \mathrm{yr}$. This indicates that the outflow has been in place for $\sim 100 \mathrm{Myr}$, which is a non-negligible fraction of the age of the Universe at this redshift (i.e. 850 Myr with the adopted cosmology).

One should note that our measurement of $\tau_{\text {dyn }}$ neglects projection effects, more specifically, the fact that the observed distance $R$ of the clumps from the centre is the projected distance, and that the observed velocity is actually only the component along our line of sight. As a consequence, the dynamical time scales should be corrected by a factor $|\tan \phi|^{-1}$, i.e. $\tau_{\text {dyn }}($ real $)=\tau_{\text {dyn }}($ obs $) /|\tan \phi|$, where $\phi$ is the angle between our line of sight and the outflow direction of each individual cloud. Obviously $\phi$ is not known. However, in the absence of any additional information on the outflow geometry, the most simplistic assumption we can make is that of a uniform distribution of the outflowing clouds' velocities as a function of $\phi$. In this case, the correction factor averaged over the entire solid angle, $\left\langle\frac{1}{|\tan \phi|}\right\rangle=\frac{1}{4 \pi} \int_{4 \pi}|\tan \phi|^{-1} \mathrm{~d} \Omega$, is equal to unity. Therefore, although the dynamical time scales may need to be corrected by a significant factor for the individual clumps, the global average trends may not be affected significantly.

\subsubsection{Integrated outflow mass-loss rate, kinetic power and momentum rate}

In order to calculate the outflow mass-loss rate, we need to estimate the mass of gas carried by the outflow. To do this, we

4 We note that in this work we also use the term "clumps" to refer to the blobs of [C II ] emission detected in outflow. The reader should be aware that these clumps or blobs generally do not correspond to individual clouds, as the minimum physical size probed by our observations (given by the synthesised beam) is $\sim 6-7 \mathrm{kpc}$. 


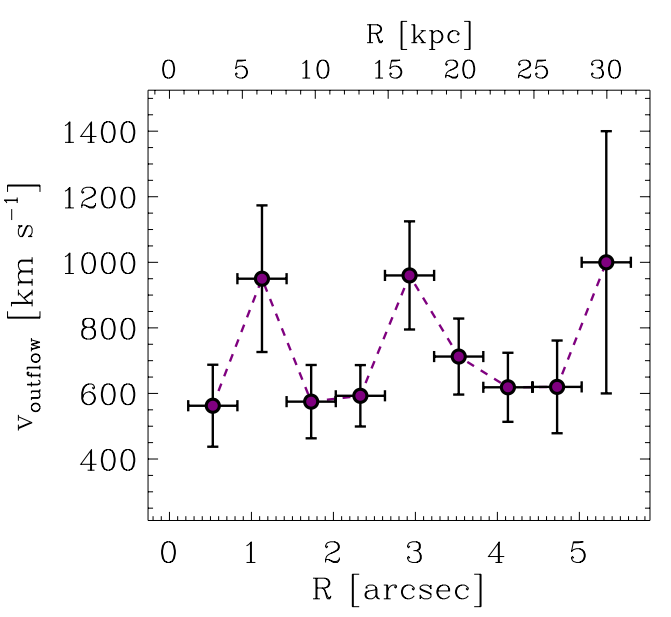

Fig. 5. Outflow velocity as a function of the radial distance from the quasar position. Both $R$ and $v$ are projected quantities. To produce this plot, the outflowing clumps have been divided into bins of radial distance from the quasar $(\Delta R=0.6 \operatorname{arcsec}$, i.e. $\simeq 3 \mathrm{kpc})$. The plot shows the average velocity of the clumps within each bin $\left(\langle v\rangle=\sum_{i=1}^{i=N} v_{i}\right.$, where $N$ is the number of clumps in each bin). The error bars in the $x$ direction indicate the bin size. The error on $\langle v\rangle$ is given by $\sigma_{\langle v\rangle}=\left(\sum_{i=1}^{i=N} \sigma_{v_{i}}^{2}\right)^{1 / 2} / N$, where $\sigma_{v_{i}}$ is the uncertainty on the average velocity $v_{i}$ of a clump in outflow, estimated as explained in Appendix C.

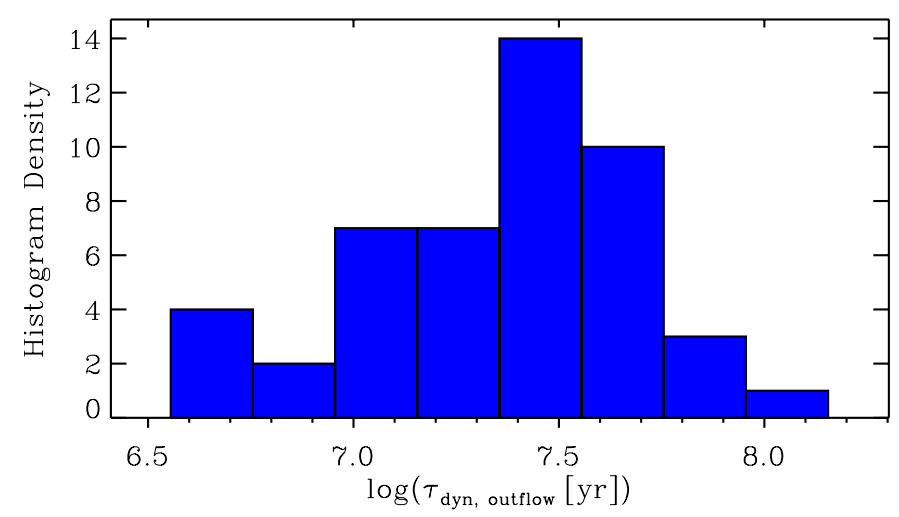

Fig. 6. Distribution of dynamical time scales $\left(\tau_{\mathrm{dyn}}=R / v\right.$, where $R$ is the projected distance from the central quasar and $v$ is the outflow velocity) within the outflow, obtained for the individual outflowing clumps as described in Sect. 3.4 and in Appendix C.

first measure the [C II] flux associated with each outflowing clump, as explained in Appendix C. The relationship between [C II] luminosity and gas mass depends on the gas density, temperature and abundance of $\mathrm{C}^{+}$ions $\left(X_{\mathrm{C}^{+}}\right)$. We convert the [C II] luminosity of the outflowing clumps into gas mass by using the formula provided by Hailey-Dunsheath et al. (2010), obtained under the hypothesis of optically thin [C II] emission. To this regard, we note that the assumption of optically thin [C II] has been challenged by observations of high-z dusty star forming galaxies (Neri et al. 2014; Gullberg et al. submitted). However, optically thick conditions for [C II] have been proposed to occur in extremely compact and dense starburst systems. It is unlikely that the diffuse and very extended [C II] outflowing component detected by us can be optically thick, even because the large velocity gradients in the outflow make self absorption even more unlikely. As in Maiolino et al. (2012), we assume that $X_{\mathrm{C}^{+}}=1.4 \times 10^{-4}, T=200 \mathrm{~K}$, and $n \gg n_{\text {crit }}$ $\left(n_{\text {crit }} \sim 3 \times 10^{3} \mathrm{~cm}^{-3}\right)$, typical of photo-dissociated regions (PDRs), and that $70 \%$ of the [C II] flux arises from such medium (while the remaining 30\% arises from other ISM phases).
The integrated outflow rate that we obtain by adding up the mass-loss rate contribution (i.e. $\dot{M}_{\text {out }}=M_{\text {out }} / \tau_{\text {dyn }}$ ) from all of the outflowing clumps is $1400 \pm 300 M_{\odot} \mathrm{yr}^{-1}$. The error on $\dot{M}_{\text {out }}$ (see Appendix $\mathrm{C}$ for the details), does not take into account the uncertainty on the conversion from [C II] luminosity to atomic gas mass. We stress that our measurement should be considered as a lower limit on the total outflow mass-loss rate, in first place because of the conservative assumptions we made for estimating the atomic gas mass from the [C II] luminosity, and in second place because it only accounts for the atomic gas phase of the outflow, while a significant amount of the mass in outflow may be in the molecular phase, similarly to local ultra-luminous infrared galaxies (ULIRGs) (e.g. Cicone et al. (2014) and refs therein).

The integrated outflow mass-loss rate measured using this "resolved" approach is over a factor of 2 lower than the previous estimate by Maiolino et al. (2012), which was based on lower resolution and lower sensitivity data and relied on a simplistic model for the outflow geometry. Conversely, our new method for estimating the outflow mass-loss rate, described in Sect. 3.4.1 and in Appendix C, does not assume any model for the outflow geometry. Furthermore, the outflow size estimated by Maiolino et al. (2012) by fitting the $u v$ data with a simple Gaussian model was only of $R \simeq 1.5 \operatorname{arcsec}(\simeq 8 \mathrm{kpc})$, i.e. half the average $\langle R\rangle \simeq 3$ arcsec that we measure by applying our "resolved" approach to the new dataset. It is therefore not surprising that our revised estimate of the outflow mass-loss rate in SDSS J1148+5251 is lower than the value that was obtained by Maiolino et al. (2012), although our estimate should be considered a lower limit as explained above.

We estimate the integrated kinetic power and momentum rate of the outflow by summing up the contribution from all outflowing clumps. Specifically, the sum of $1 / 2 \dot{M}_{\text {out }} v^{2}$ and $\dot{M}_{\text {out }} v$, evaluated separately for each blob in outflow, gives, respectively, an integrated outflow kinetic power of $(3.3 \pm 0.5) \times 10^{44} \mathrm{erg} \mathrm{s}^{-1}$ and an integrated outflow momentum rate of $(7.2 \pm 1.0) \times$ $10^{36} \mathrm{~g} \mathrm{~cm} \mathrm{~s}^{-2}$. It is common practice in studies of AGN-driven outflows to compare these quantities with the AGN bolometric luminosity $\left(L_{\mathrm{AGN}}\right)$ and with the total radiative momentum output from the AGN $\left(L_{\mathrm{AGN}} / c\right)$. In this case, by using the intrinsic AGN luminosity of $L_{\mathrm{AGN}}=5.63 \times 10^{13} L_{\odot}$ inferred by Schneider et al. (2014) for SDSS J1148+5251, the ratio between our estimate of the outflow kinetic power and $L_{\mathrm{AGN}}$ is $(1.6 \pm 0.2) \times 10^{-3}$, and the ratio between the momentum rate and $L_{\mathrm{AGN}} / c$ is $1.00 \pm 0.14$. These values and their implications will be discussed in Sect. 4.1. We also discuss in Sect. 4.1 the possible contribution of star formation to driving the observed outflow.

\subsubsection{Outflow properties as a function of $\tau_{\text {dyn }}$ and $R$}

In Sect. 3.4.2 we have derived the integrated outflow mass-loss rate, kinetic power and momentum rate. However, one may argue that given the quite broad distribution in dynamical time scales within the outflow evidenced in Fig. 6, the clumps were not ejected all at the same time as a consequence of a single, explosive event, but, instead, they may have been expelled from the galaxy at different epochs. It is therefore interesting to investigate the contribution to the mass-loss rate, kinetic power and momentum rate of the outflow from clumps with different "ages", i.e. with different $\tau_{\text {dyn }}$. To this end, we present in Fig. 7 the massloss rate, the kinetic power ratio $\left(1 / 2 \dot{M}_{\text {out }} v^{2}\right) / L_{\mathrm{AGN}}$ and momentum rate ratio $\left(\dot{M}_{\text {out }} v\right) /\left(L_{\mathrm{AGN}} / c\right)$ of the outflow as a function of 
C. Cicone et al.: Very extended cold gas, star formation and outflows in the halo of a bright quasar at $z>6$
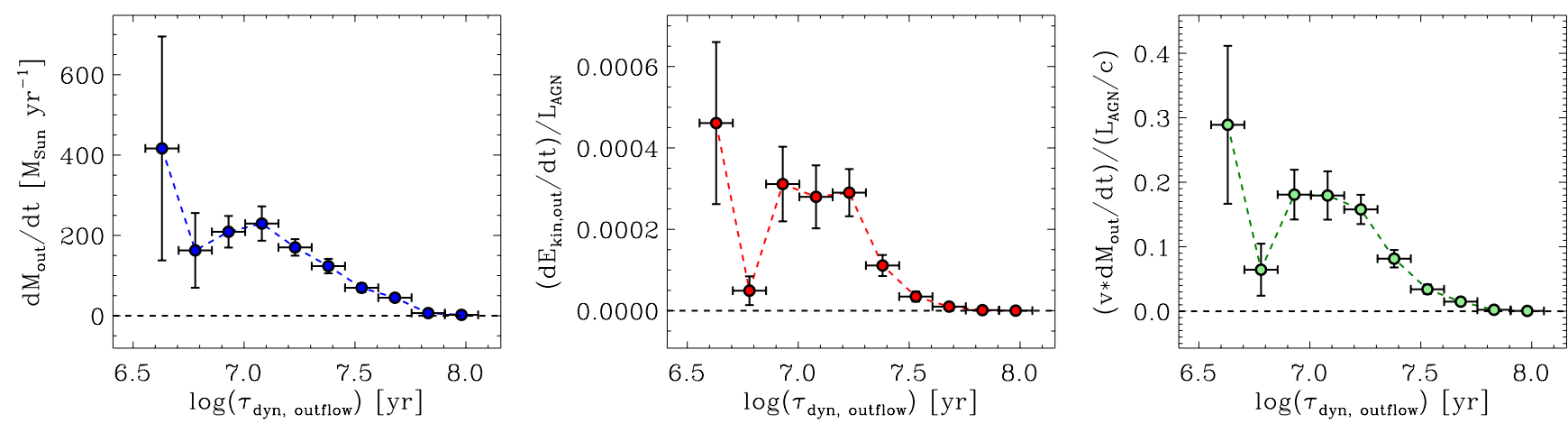

Fig. 7. Outflow mass-loss rate $\dot{M}_{\text {out }}($ left $)$, kinetic power ratio $\left(1 / 2 \dot{M}_{\text {out }} v^{2}\right) / L_{\mathrm{AGN}}($ centre $)$ and momentum rate ratio $\left(\dot{M}_{\text {out }} v\right) /\left(L_{\mathrm{AGN}} / c\right)($ right $)$ as a function of the dynamical time scale of the clumps in outflow. These plots have been obtained by dividing the outflowing clumps (see Sect. 3.4) in bins of $\Delta \log \left(\tau_{\text {dyn }}\right)=0.15$. The plots show, in correspondence of the average $\tau_{\text {dyn }}$ of each bin, the total $\dot{M}_{\text {out }},\left(1 / 2 \dot{M}_{\text {out }} t^{2}\right) / L_{\text {AGN }}$ and $\left(\dot{M}_{\text {out }} v\right) /\left(L_{\mathrm{AGN}} / c\right)$ obtained by adding up the contribution of all the outflowing clumps within the bin. The error bars in the $x$ direction indicate the bin size, i.e. $\Delta x= \pm 0.075$. The error bars in the $y$ direction are calculated using the statistical error propagation (i.e. by propagating the errors on $v, R$ and on the [C II] fluxes measured for each clump in outflow, which are estimated in Appendix C). The errors do not take into account the uncertainty on the conversion from [C II] luminosity to atomic gas mass and the uncertainty on the AGN luminosity estimate (Schneider et al. 2014).
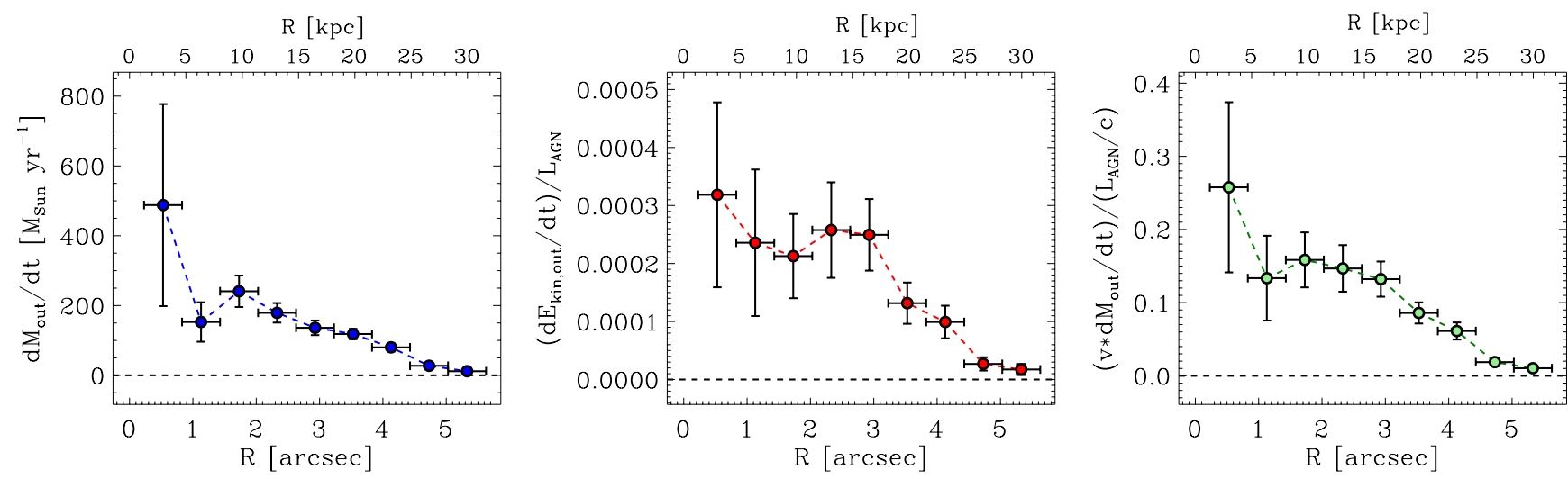

Fig. 8. Outflow mass-loss rate $\dot{M}_{\text {out }}($ left $)$, kinetic power ratio $\left(1 / 2 \dot{M}_{\text {out }} v^{2}\right) / L_{\mathrm{AGN}}($ centre $)$ and momentum rate ratio $\left(\dot{M}_{\text {out }} v\right) /\left(L_{\mathrm{AGN}} / c\right)(r i g h t)$ as a function of the projected distance from the quasar position. These plots have been obtained by dividing the outflowing clumps (see Sect. 3.4) in bins of $\Delta R=0.6 \mathrm{arcsec}$, corresponding to $\simeq 3 \mathrm{kpc}$. The plots show, in correspondence of the average $R$ of each bin, the total $\dot{M}_{\text {out }},\left(1 / 2 \dot{M}_{\text {out }} v^{2}\right) / L_{\mathrm{AGN}}$ and $\left(\dot{M}_{\text {out }} v\right) /\left(L_{\mathrm{AGN}} / c\right)$ obtained by adding up the contribution of all the outflowing clumps within the bin. For an explanation of the error bars, see caption of Fig. 7.

the dynamical time scale of the outflowing clumps. Figure 7 suggests that the ejection of gas has not occurred at a constant rate in SDSS J1148+5251. In particular, the most extreme outflow event seems to have happened more recently, within the past $\sim 5 \mathrm{Myr}$, as indicated by the first prominent peak of outflow mass-loss rate, kinetic power and momentum flux in Fig. 7. Interestingly, Fig. 7 shows the presence of a second, broader peak of these outflow properties between $\tau_{\text {dyn }} \in(8-20)$ Myr. Taken at face value, these findings would imply that, although the outflow may have been in place for $100 \mathrm{Myr}$, the bulk of the mass, energy and momentum have been released throughout two major outflow events happened more recently, within the past 20 Myr (Fig. 7).

A similar non-uniform distribution of the outflow mass-loss rate, kinetic power and momentum rate is also evidenced as a function of the (projected) distance from the quasar, as shown by Fig. 8, in which we report the radial profiles of the outflow properties. Figure 8 indicates that, overall, $\dot{M}_{\text {out }},\left(1 / 2 \dot{M}_{\text {out }} v^{2}\right) / L_{\mathrm{AGN}}$ and $\left(\dot{M}_{\text {out }} v\right) /\left(L_{\mathrm{AGN}} / c\right)$ decrease with the distance from the quasar. The main peak can be identified at projected radial distance of $\sim 0.5$ arcsec from the position of the central quasar, corresponding to $\sim 3 \mathrm{kpc}$. There is also marginal evidence for a second, broad peak between $r \in(1.5-3)$ arcsec (i.e. $r \in(8-17) \mathrm{kpc})$. Similarly to what suggested for Fig. 7, the two peaks in Fig. 8 may be associated to major outflow events during the recent life of the quasar-galaxy. However, the significance of the second peak at $r \in(8-17) \mathrm{kpc}$ is low, so this interpretation should be taken with caution. Figure 8 can be readily compared with the predictions of recent theoretical studies of AGN feedback (Costa et al. 2014b; Thompson et al. 2014). This discussion is presented in Sect. 4.1.

We stress that observations are naturally limited for large $R$ (and hence for high $\tau_{\mathrm{dyn}}$ ): indeed, outflow clumps at large distances from the galaxy centre are more difficult to probe due to the sensitivity of our maps. The lowest outflow velocities (which can also give high values of $\tau_{\text {dyn }}$ ) are also difficult to probe due to contamination by quiescent gas, i.e. the narrow component of the [C II] line. In addition, "old" outflowing clumps, associated with longer dynamical time scales and typically at larger distances, are more likely to have dissolved or evaporated. Therefore, the decreasing outflow rate with $\tau_{\text {dyn }}$ and radius may be in part the result of sensitivity issues in the observations and/or of the effect of outflowing clouds dissolving as they travel to larger distances, or being decelerated by the interaction with the intergalactic and circumgalactic media. For similar reasons, it is difficult to assess with the current data whether the outflow in SDSS J1148+5251 

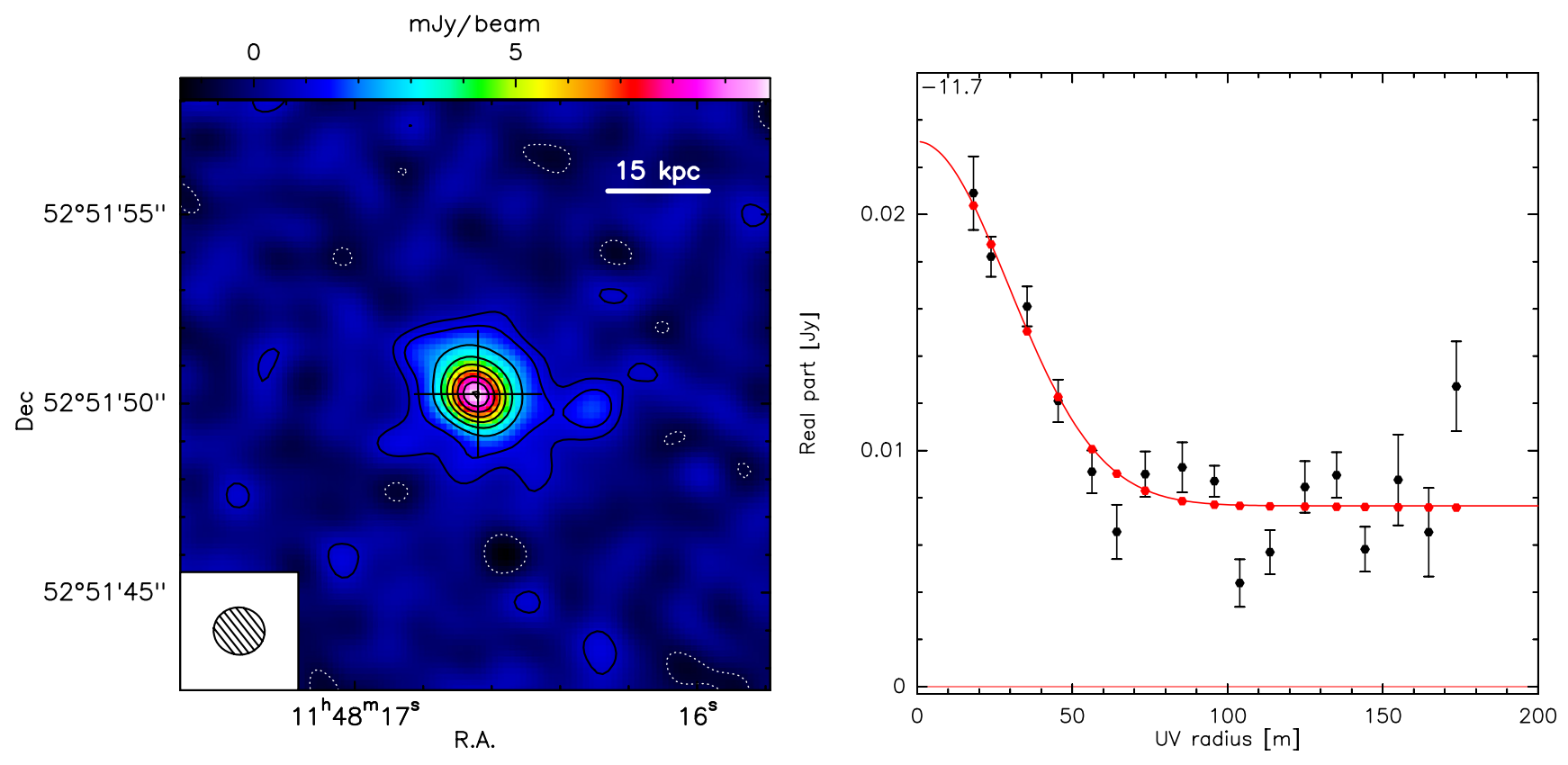

Fig. 9. IRAM PdBI continuum-subtracted map (left) and $u v$ plane analysis (right) of the core of the [C II] $158 \mu \mathrm{m}$ emission line of SDSS J1148+5251, integrated within $v \in(-200,200) \mathrm{km} \mathrm{s}^{-1}$. Left: negative and positive contours correspond to $-3 \sigma, 3 \sigma, 5 \sigma$ to $40 \sigma$ in steps of $5 \sigma\left(1 \sigma \mathrm{rms}\right.$ noise is $\left.0.10 \mathrm{Jy}_{\text {beam }}^{-1} \mathrm{~km} \mathrm{~s}^{-1}\right)$. The cross indicates the pointing and phase centre, corresponding to the optical position of the quasar. Right: real part of visibilities as a function of the $u v$ distance ( $u v$ plot), binned in baseline steps of $10 \mathrm{~m}$. The red curve indicates the best fit to the $u v$ plot, which corresponds to the sum of two components: a point (unresolved) source, responsible for the flattening of the visibilities at large $u v$ radii $(u v$ radii $>50 \mathrm{~m}$ ), and an extended (resolved) source fitted with a circular Gaussian model, which is responsible for the sharp decline of the visibilities at small $u v$ radii.

manages to escape the viral radius of the host galaxy and to enrich the intergalactic medium (IGM).

\subsection{Quiescent (narrow) [CII] emission}

The map of the narrow component of the [C II] $158 \mu \mathrm{m}$ emission, integrated within $v \in(-200,200) \mathrm{km} \mathrm{s}^{-1}$, is presented in Fig. 9. The source is resolved at high significance by our PdBI observations, and it appears surprisingly extended, on scales $\gtrsim 20 \mathrm{kpc}$. This could already be inferred from the spectra extracted from different apertures, which showed that the flux of the narrow component is a factor of $\sim 2$ larger in the 4 arcsec-aperture spectrum with respect to the 2 arcsec-aperture spectrum (Fig. 1 and Table 1).

The $u v$ plot of the narrow [C II] $158 \mu \mathrm{m}$ emission, in the right panel of Fig. 9, provides important information about the structure of the source. In particular, the $u v$ plot clearly shows the presence of two components: a compact component (not resolved), which is responsible for the flattening of the $u v$ visibility amplitude at large $(>50 \mathrm{~m}) u v$ radii, and an extended component (resolved at high significance), which is at the origin of the decreasing trend of the $u v$ visibility amplitude with radius at small $u v$ radii $^{5}$. The red curve represents our best fit model, which is obtained by fitting the compact component with a point source and the resolved component with a circular Gaussian distribution. The resulting FWHM size of the circular Gaussian model is $3.1 \pm 0.3$ arcsec, corresponding to $17.4 \pm 1.7 \mathrm{kpc}$. The velocity-integrated fluxes derived from the $u v$ modelling are $6.2 \pm 0.6 \mathrm{Jy} \mathrm{km} \mathrm{s}{ }^{-1}$ and $3.08 \pm 0.12 \mathrm{Jy} \mathrm{km} \mathrm{s}{ }^{-1}$ for the resolved/extended and

\footnotetext{
5 We note that the detection of this spatially extended component has also been confirmed by splitting the dataset into independent parts.
}

point sources, respectively. These fluxes can be translated into [C II] luminosities of $L_{[\mathrm{CII}] \text {,ext }}=(6.7 \pm 0.7) \times 10^{9} L_{\odot}$ and $L_{[\mathrm{CII}] \text {,point }}=(3.33 \pm 0.13) \times 10^{9} L_{\odot}$. Strikingly, the $u v$ fit indicates that about $70 \%$ of the total $[\mathrm{C} \mathrm{II}]$ emission at velocities $v \in(-200,200) \mathrm{km} \mathrm{s}^{-1}$ is in the extended component. We note that, however, the quality of the data does not allow us to distinguish between different models for the extended source. In particular, an exponential intensity distribution would also provide a good fit to the resolved component.

The extended and compact components contributing to the narrow [C II] emission within $v \in(-200,200) \mathrm{km} \mathrm{s}^{-1}$ can be disentangled in the $u v$ plane and, therefore, imaged separately, by using the model fit shown in Fig. 9. In the upper panel of Fig. 10 we show the map of the extended component, obtained by subtracting from the $u v$ data the point source model fit. The map shows an irregular ring-like and filamentary structure, with the most prominent filament extending up to $r \sim 28 \mathrm{kpc}(5 \operatorname{arcsec})$ in the western direction with respect to the quasar position. The centroid of the extended source is slightly shifted to the southeast with respect to the optical quasar position (indicated by the cross). The map of the compact component is shown in the bottom panel of Fig. 10, and it has been obtained by applying a $u v$ tapering that cuts the visibilities with $u v$ radius $<80 \mathrm{~m}$. This bright, compact source, unresolved in our data and peaking at the quasar position, had already been detected and imaged by Walter et al. (2009) and it is likely tracing a kpc-scale hyper-starburst in the centre of SDSS J1148+5251.

We find no evidence for a regular rotation pattern in our IRAM PdBI [C II] maps (Appendix D). A spatial shift between the blue- and the red-shifted sides of the [C II] line was found in previous high resolution interferometric [C II] observations by Walter et al. (2009), which we now know that were resolving out a large portion of extended [C II] flux ( $\sim 70 \%$ within 

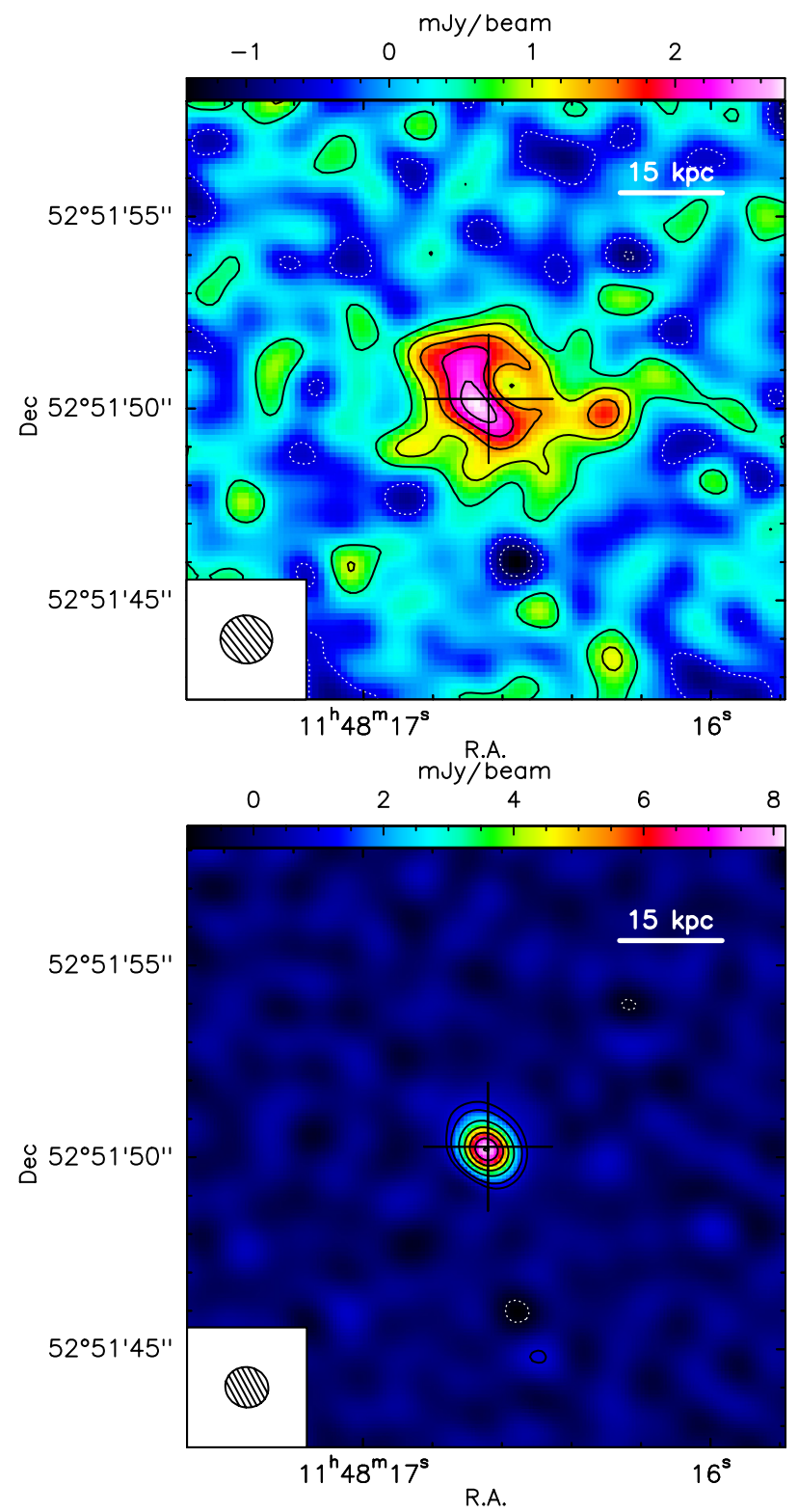

Fig. 10. Maps of the extended (top) and compact (bottom) components that contribute to the core [C II] $158 \mu \mathrm{m}$ emission of SDSS J1148+5251 within $v \in(-200,200) \mathrm{km} \mathrm{s}^{-1}$. The map of the extended component has been obtained by subtracting from the $u v$ data the point source model fit. The map of the compact component has been produced by applying a $u v$ tapering that cuts the visibilities with $u v$ radius $<80 \mathrm{~m}$; this operation increases the spatial resolution of the data (the resulting beam size is $\left.1.1^{\prime \prime} \times 1.1^{\prime \prime}\right)$. Negative and positive contours are in steps of $2 \sigma$ for the extended component in the top panel ( $1 \sigma \mathrm{rms}$ noise of $0.10 \mathrm{Jy} \mathrm{beam}^{-1} \mathrm{~km} \mathrm{~s}^{-1}$ ). For the compact component in the bottom panel, contours correspond to $-3 \sigma, 3 \sigma, 5 \sigma$ to $30 \sigma$ in steps of $5 \sigma$ $\left(1 \sigma\right.$ rms noise of $\left.0.11 \mathrm{Jy} \mathrm{beam}^{-1} \mathrm{~km} \mathrm{~s}^{-1}\right)$. In both panels, the cross indicates the pointing and phase centre, corresponding to the optical position of the quasar.

$v \in(-200,200) \mathrm{km} \mathrm{s}^{-1}$ as shown by our $u v$ fit in Fig. 9). While the compact source, which is not resolved by our observations, is probably rotationally-broadened as suggested by Walter et al. (2009), the hypothesis of rotation does not seem to hold for the dominant extended component, as shown in Fig. D.1, although some irregular velocity pattern is observed, possibly associated with some minor mergers. This implies that it is difficult to estimate the dynamical mass of this system on large scales.
The spatially extended and kinematically quiescent (i.e. "narrow") [C II] source discovered in SDSS J1148+5251 (top panel of Fig. 10) is reminiscent of the giant $(r \gtrsim$ $50 \mathrm{kpc}$ ) low surface brightness and kinematically unperturbed $\left(\sigma_{v} \lesssim 300 \mathrm{~km} \mathrm{~s}^{-1}\right)$ haloes found in high redshift radio galaxies (HzRGs) by Villar-Martín et al. (2003). These quiescent nebulae around HzRGs are detected in several emission lines (Ly $\alpha$, $\mathrm{C} \mathrm{IV}, \mathrm{He}$ II, N V) and are thought to trace giant halo gas reservoirs (rather than companion galaxies) photoionised primarily by the radio-loud quasar continuum. Although SDSS J1148+5251 is not radio-loud (Carilli et al. 2004), and although previous Hubble Space Telescope (HST) observations by Decarli et al. (2012) did not find evidence for extended Ly $\alpha$ emission (possibly due to strong dust extinction on large scales, as supported by our FIR continuum observations described in Sect. 3.6), our new IRAM PdBI observations of an extended and quiescent [C II] halo surrounding this high- $z$ quasar show some similarities (e.g. the spatial extent and the velocity dispersion) with the gas haloes surrounding HzRGs. In the case of SDSS J1148+5251, however, we also detect associated FIR continuum emission up to $r \simeq 8-10 \mathrm{kpc}$ (Sect. 3.6), suggesting that extended star formation, rather than the quasar, is the main excitation source of the [C II] halo, at least up to scales of $r \simeq 10 \mathrm{kpc}$. We further discuss the origin of the extended [C II] source and its connection with star formation in Sects. 4.2 and 4.3.

\subsection{Analysis of the FIR continuum extent}

We investigate the presence of extended dust emission in SDSS J1148+5251 by using ancillary high sensitivity IRAM PdBI observations of the FIR continuum in this source obtained as a part of a parallel project presented by Gallerani et al. (2014) (see also Sect. 2). These deep ancillary continuum observations at $262 \mathrm{GHz}$ and $259.4 \mathrm{GHz}$ are particularly suited for studying the extent of the FIR emission in SDSS J1148+5251, as, once merged, they reach a continuum sensitivity of $0.082 \mathrm{mJy}^{\text {beam }}{ }^{-1}$ and have a slightly higher spatial resolution (synthesised beam of $\left.1.1^{\prime \prime} \times 1.0^{\prime \prime}\right)$ than our main dataset at $256 \mathrm{GHz}$.

The left panel of Fig. 11 shows the map of the FIR continuum emission of SDSS $\mathrm{J} 1148+5251$ at an average rest-frame wavelength of $\lambda=155 \mu \mathrm{m}$ (average observed frequency of $261 \mathrm{GHz}$ ), produced by merging the two datasets at $262 \mathrm{GHz}$ and $259.4 \mathrm{GHz}$ as explained in Sect. $2^{6}$. The corresponding $u v$ visibility diagram is shown in the right panel of Fig. 11. Similarly to the narrow [C II] $158 \mu \mathrm{m}$ emission (Fig. 9), the dust thermal emission can also be disentangled in the $u v$ data into a compact (unresolved) and an extended (resolved) component. Accordingly, we fit the $u v$ plot of the $155 \mu \mathrm{m}$ FIR continuum with a combination of a point source model and a circular Gaussian model, and show the best fit as a red curve in Fig 11. We stress that, although the circular Gaussian model provides a good fit to the data, it constitutes a simplistic approximation, and better quality data may reveal a more complex structure. The $u v$ fit indicates that $45 \pm 9 \%$ of the FIR emission at this wavelength is in the extended source, and it provides a FWHM size for the circular Gaussian component of $2.0 \pm 0.5$ arcsec, corresponding to $11 \pm 3 \mathrm{kpc}$. Taken at face value, this result suggests

\footnotetext{
6 A zoomed-out version of this map, showing the marginal detection of an additional continuum source in the field, is shown in Fig. A.1 (Appendix A). In Fig. A.1 we also show a map of the $256 \mathrm{GHz}$ continuum, obtained by collapsing the line-free channels of the main dataset and used for the purpose of continuum-subtraction as described in Sect. 3.1.
} 

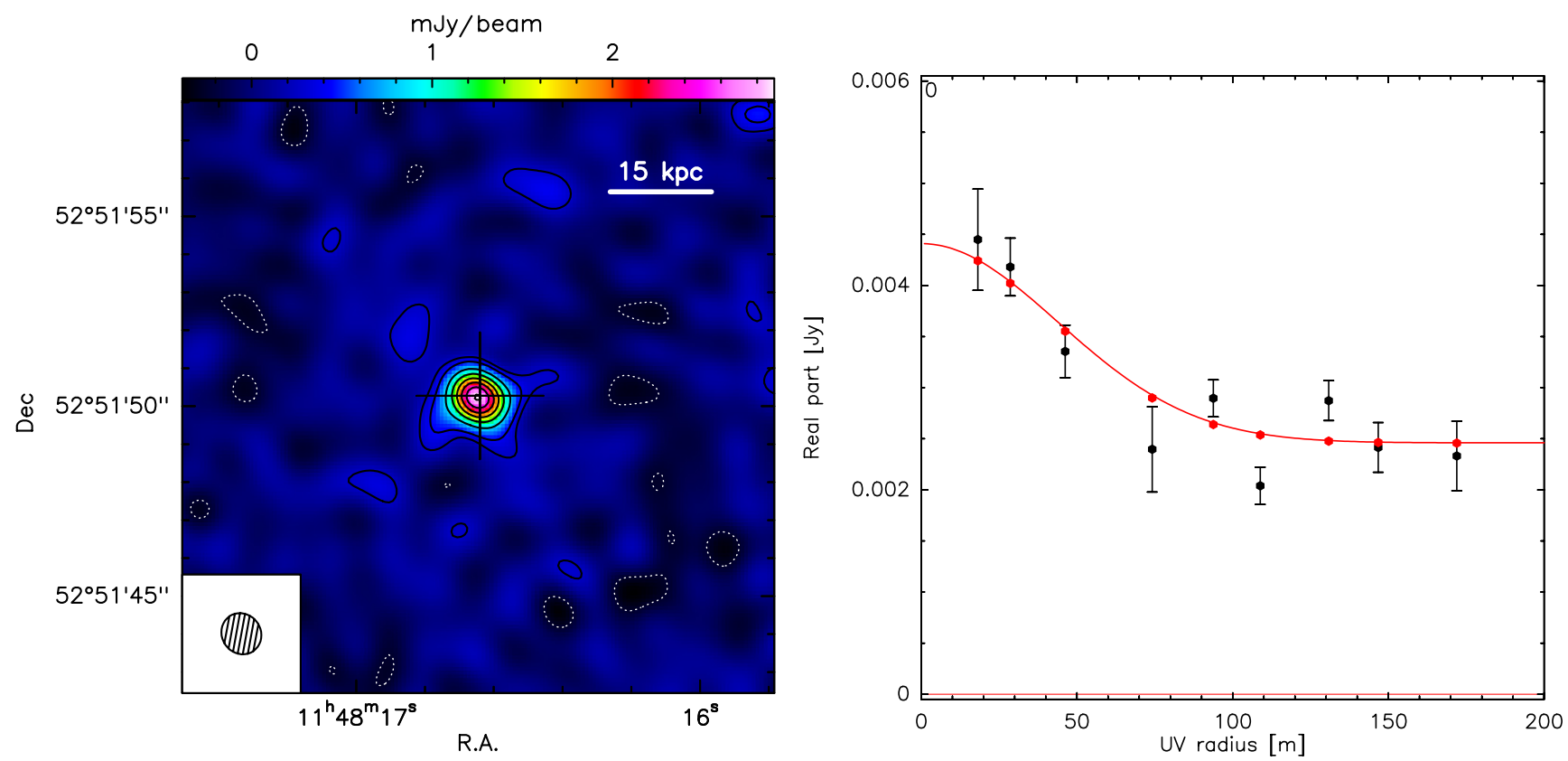

Fig. 11. IRAM PdBI map (left) and $u v$ plane analysis (right) of the FIR continuum of SDSS J1148+5251 at average rest-frame wavelength $\lambda=155 \mu \mathrm{m}$ (average observed frequency of $261 \mathrm{GHz}$ ). Left: negative and positive contours correspond to $-3 \sigma, 3 \sigma, 5 \sigma$ to $35 \sigma$ in steps of $5 \sigma$ $\left(1 \sigma \mathrm{rms}\right.$ noise is $\left.0.082 \mathrm{mJy}_{\text {beam }}^{-1}\right)$. The synthesised beam is shown at the bottom left corner $\left(1.1^{\prime \prime} \times 1.0^{\prime \prime}\right)$ and the cross indicates the pointing and phase centre, corresponding to the optical position of the quasar. Right: real part of visibilities as a function of the $u v$ distance ( $u v$ plot), binned in baseline steps of $20 \mathrm{~m}$. The red curve indicates the best fit to the $u v$ plot, which corresponds to the sum of two components: a point (unresolved) source, responsible for the flattening of the visibilities at large $u v$ radii ( $u v$ radii $>100 \mathrm{~m}$ ), and an extended (resolved) source fitted with a circular Gaussian model, which is responsible for the decreasing trend at smaller $u v$ radii.

that the narrow $[\mathrm{C} \mathrm{II}](F W H M=17.4 \pm 1.7 \mathrm{kpc})$ extends beyond the region of intensive and high surface density star formation in the host galaxy of SDSS J1148+5251, traced by the FIR continuum emission $(F W H M=11 \pm 3 \mathrm{kpc})$ (further discussion in Sect. 4).

We use the $u v$ fit results for producing separate maps of the extended and compact FIR sources, which are presented in Fig. 12. Strikingly, the resolved component exhibits a tail extending by $r \sim 10 \mathrm{kpc}$ in the western direction, i.e. the same direction as the $r \sim 28 \mathrm{kpc}$ filament observed in the map of the extended [C II] (Fig. 10). This suggests that the western [C II] filament embeds star formation heating dust on $r \sim 10 \mathrm{kpc}$ scales.

In order to estimate the FIR luminosity associated with the extended and compact dust continuum sources, we apportion the total FIR luminosity of SDSS J1148+5251, $L_{\mathrm{FIR}(42.5-122.5 \mu \mathrm{m})}=$ $(1.3 \pm 0.2) \times 10^{13} L_{\odot}\left(\right.$ Rosa Valiante, priv. comm. $\left.{ }^{7}\right)$, according to the flux ratio estimated from our $u v$ modelling of the FIR emission at $155 \mu \mathrm{m}$ (Fig. 11). This results into FIR $(42.5-122.5 \mu \mathrm{m})$ luminosities of $L_{\mathrm{FIR}, \mathrm{ext}}=(6.0 \pm 1.5) \times 10^{12} L_{\odot}$ and $L_{\text {FIR,point }}=(7.4 \pm 1.6) \times 10^{12} L_{\odot}$ for the extended and compact component, respectively.

\section{Discussion}

\subsection{The driving mechanism of the outflow}

In Sect. 3.4 we have studied with unprecedented detail the properties of the massive and extended [C II] outflow of SDSS J1148+5251. We have shown that the [C II]-emitting gas

\footnotetext{
7 This value of $L_{\mathrm{FIR}(42.5-122.5 \mu \mathrm{m})}$ has been estimated by using the same method as in Valiante et al. (2011), by restricting the integration range to the wavelength range $42.5-122.5 \mu \mathrm{m}$.
}

in this source reaches extremely high projected outflow velocities of $v \sim 1400 \mathrm{~km} \mathrm{~s}^{-1}$, with an average outflow velocity of $v \simeq 800 \mathrm{~km} \mathrm{~s}^{-1}$ (Fig. 5). The observed very high velocities strongly favour the AGN as the dominant driving mechanism of the outflow in SDSS J1148+5251.

However, SDSS J1148+5251, besides hosting a powerful unobscured quasar $\left(L_{\mathrm{AGN}}=5.63 \times 10^{13} L_{\odot}\right.$, Schneider et al. 2014), is also a powerful starbursting galaxy (Walter et al. 2009). In particular, our new deep FIR continuum observations show the presence of strong and spatially extended warm dust continuum emission, indicative of intense star formation occurring up to scales of at least $r \sim 8-10 \mathrm{kpc}$ (see also discussion in Sect. 4.3). By splitting the total infrared (TIR, 8-1000 $\mu \mathrm{m}$ ) luminosity of $L_{\operatorname{TIR}(8-1000 \mu \mathrm{m})}=(2.2 \pm 0.3) \times 10^{13} L_{\odot}$ (Valiante et al. 2011) according to the flux ratio between extended and compact component estimated from our $u v$ analysis of the FIR continuum emission (Sect. 3.6), we obtain for the extended component $L_{\mathrm{TIR}, \text { ext }}=(10 \pm 2) \times 10^{12} L_{\odot}$. By using the relation in Kennicutt (1998), a Chabrier IMF and a negligible AGN contribution (such assumption is only valid for the extended component, as the AGN contribution to dust heating may be significant in the unresolved component, as shown by Schneider et al. 2014), the TIR luminosity can be converted into a star formation rate of $S F R_{\mathrm{ext}}=(1000 \pm 200) M_{\odot} \mathrm{yr}^{-1}$. Supernova events associated with such an high star formation rate may also be an important source of feedback in SDSS J1148+5251 (see also Costa et al. 2014c,a). Following Veilleux et al. (2005), the kinetic power injected by supernovae associated with $\mathrm{SFR}_{\mathrm{ext}}$ is $P_{\text {kin,SF }}=(7.0 \pm 1.7) \times 10^{44} \mathrm{erg} \mathrm{s}^{-1}$. A comparison between $P_{\text {kin,SF }}$ and our (lower limit) estimate of the integrated outflow kinetic power of $P_{\text {kin,outflow }}=(3.3 \pm 0.5) \times 10^{44} \mathrm{erg} \mathrm{s}^{-1}($ Sect. 3.4.2) would give a lower limit on the coupling efficiency (by assuming a purely star formation-driven outflow) of $47 \pm 13 \%$. Such lower 


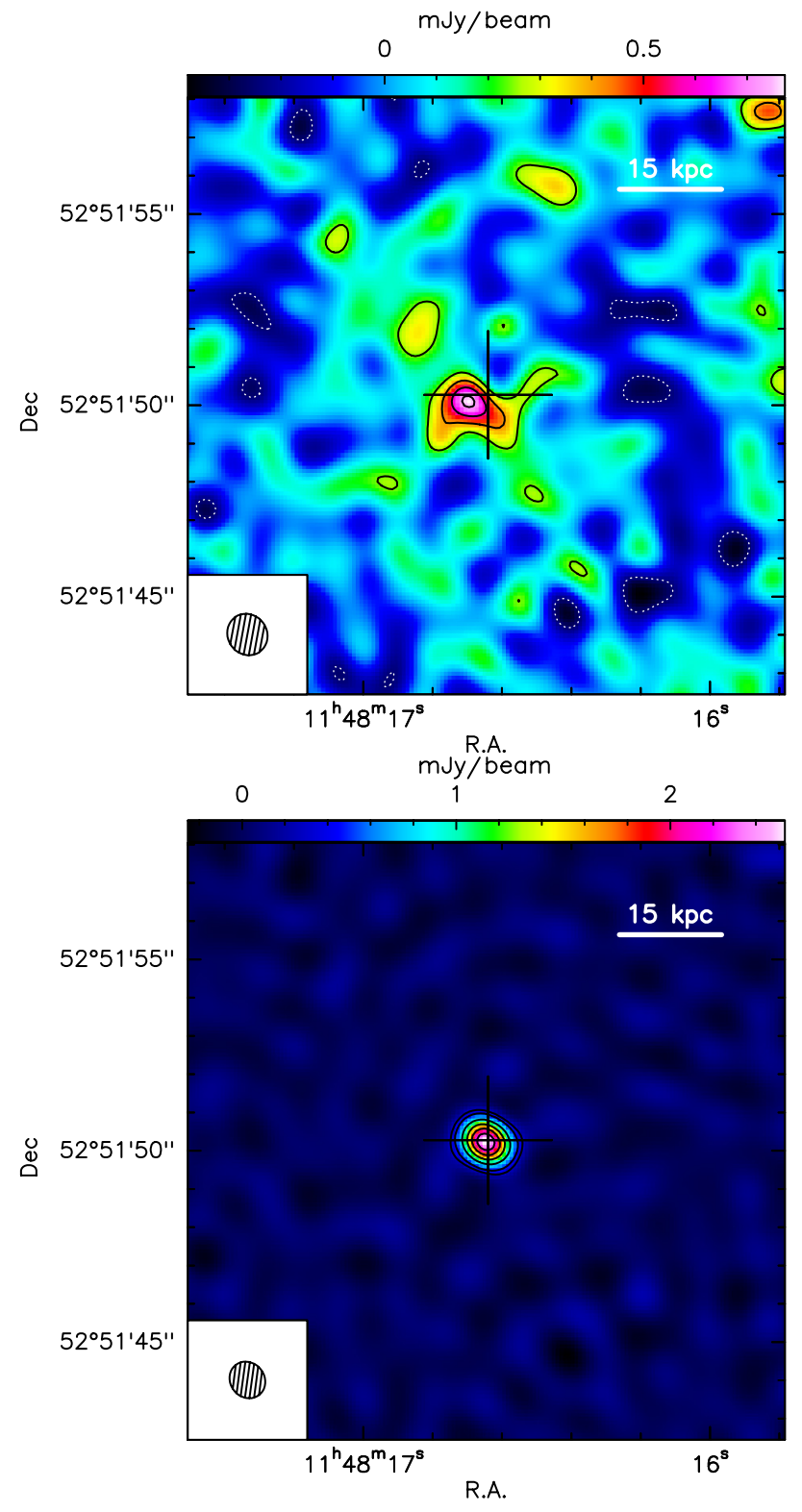

Fig. 12. Maps of the extended (top) and compact (bottom) components that contribute to the FIR continuum emission of SDSS J1148+5251 at average rest-frame wavelength $\lambda=155 \mu \mathrm{m}$. The map of the extended component has been obtained by subtracting, from the $u v$ data, the point source model of the compact component resulting from the $u v$ fit reported in Fig. 11. The map of the compact component has been obtained by applying a $u v$ tapering that cuts visibilities with $u v$ radius $<100 \mathrm{~m}$, therefore slightly increasing the resolution of the data (the resulting beam size is $0.99^{\prime \prime} \times 0.89^{\prime \prime}$ ). In the map of the extended component, contours correspond to $-3 \sigma, 3 \sigma, 5 \sigma$ to $9 \sigma$ in steps of $2 \sigma$ $\left(1 \sigma \mathrm{rms}\right.$ noise is $0.082 \mathrm{mJy}$ beam $\left.^{-1}\right)$. For the compact component, contours correspond to $-3 \sigma, 3 \sigma, 5 \sigma$ to $25 \sigma$ in steps of $5 \sigma$ ( $1 \sigma$ rms noise is $0.088 \mathrm{mJy}^{\text {beam }}{ }^{-1}$ ). In both panels, the cross indicates the pointing and phase centre, corresponding to the optical position of the quasar.

limit efficiency is already significantly higher than the value of $1-10 \%$ expected for star formation-driven winds (e.g. Cicone et al. 2014).

In the following, based on the velocity and coupling efficiency arguments, we will assume that, although the intense star formation on large scales may also play a role, the AGN gives the dominant contribution to driving the outflow. This is also in agreement with the hierarchical semianalytic models by Valiante et al. (2012), who predicted quasar feedback to be the dominant mechanism in SDSS J1148+5251. We will now discuss the properties of the major [C II] outflow in SDSS J1148+5251 in the light of current models of AGN feedback.

The models that have been proposed so far for explaining AGN-driven kpc-scale outflows can be branched into two main categories: the so-called "blast wave" models, in which large scale outflows are generated by hydrodynamical coupling of the AGN with the galaxy ISM through fast winds originating from the accretion disk (e.g. King 2003, 2005, 2010; Lapi et al. 2005; Menci et al. 2006, 2008; Zubovas \& King 2012, 2014; Faucher-Giguère \& Quataert 2012; Costa et al. 2014b and references therein), and the models invoking radiation pressure on dusty clouds as the main mechanism at the origin of galacticscale outflows (e.g. Hopkins \& Elvis 2010; Fabian 2012; Roth et al. 2012; Thompson et al. 2014 and refs therein). In the recent years, both classes of models have been tested against observations of AGN-driven outflows and, in particular, of massive kpcscale outflows of cold and dense molecular gas in nearby galaxies (Feruglio et al. 2010, 2013a,b; Alatalo et al. 2011; Sturm et al. 2011; Aalto et al. 2012; Veilleux et al. 2013; Spoon et al. 2013; Combes et al. 2013; Cicone et al. 2012, 2014; GarcíaBurillo et al. 2014). A recent study by Cicone et al. (2014) showed that the "blast wave" scenario and, specifically, the "energy-conserving" nature of the observed large-scale molecular outflows is favoured when considering a consistent sample of (U)LIRGs and powerful quasars in the local Universe. However, there are only a few studies investigating the feedback mechanisms in high-z sources and, in particular, the detail of information provided by our new [C II] observations of SDSS J1148+5251 is unprecedented. In this section we discuss our results, presented in Sect. 3.4, in the light of the proposed theoretical models for AGN-driven outflows.

In this context, theoretical works have shown that there are three main properties of outflows, namely the mass-loss rate, the momentum rate (or momentum flux) and the kinetic power, that can be used as "key" indicators for the dominant feedback mechanism in action. In Sects. 3.4 and Appendix C we have used our new [C II] observations for inferring these properties (both integrated and as a function of dynamical time scale and radius) for the very extended outflow in SDSS J1148+5251. Bearing in mind that these are lower limits (because of the caveats discussed in Sect. 3.4 and Appendix C), the integrated values that we have obtained, do not help much in discriminating between different models. In particular, the modest integrated momentum rate of $(1.00 \pm 0.14) L_{\mathrm{AGN}} / c$ and kinetic power of $(1.6 \pm 0.2) \times 10^{-3} L_{\mathrm{AGN}}$ would be in principle consistent with any of the proposed models. However, the unprecedented spatial extent of the outflow in SDSS J1148+5251 has allowed us, for the first time, to go beyond the integrated values by investigating the dependence of the outflow properties as a function of the dynamical time scale of the outflow and of the distance from the quasar (Figs. 7 and 8).

The plots in Fig. 8, in particular, allow a direct comparison with the predictions of two recent studies, Costa et al. (2014b), who incorporate the sub-grid physics representing the "blast wave" feedback prescriptions into cosmological simulations, and Thompson et al. (2014), who further develop the physics of dusty radiation-pressure driven shells and discuss it in the context of the most recent observational results. Our results appear qualitatively inconsistent with the results obtained by Costa et al. (2014b) from cosmological simulations of energy-driven outflows generated by inner accretion disk winds, which instead show that, on the same physical scales of $\$ 30$ (proper) kpc, the momentum flux and kinetic power of the outflow overall 
increase with radial distance from the AGN (Fig. 15 of Costa et al. 2014b). Moreover, quantitatively, the outflow momentum rate and kinetic power inferred for SDSS J1148+5251 are about one order of magnitude lower than the integrated values reported by Costa et al. (2014b) for the same simulation. We note however that the previously mentioned results by Costa et al. (2014b) were obtained using a central black hole with mass of $\sim 10^{8} M_{\odot}$, i.e. significantly lower than the black hole mass of $\sim 3 \times 10^{9} M_{\odot}$ estimated for SDSS J1148+5251 (Willott et al. 2003). Simulations may produce significantly different results for more massive haloes and BHs. For example, Costa et al. (2014b) show that, in presence of a more massive BH $\left(M_{\mathrm{BH}} \sim\right.$ $\left.10^{9} M_{\odot}\right)$, even in the "momentum-driven" limit, i.e. the limit in which the thermal energy input from the inner wind is not conserved, efficient large scale AGN-driven outflows may develop, comparable to those developed under "energy-conserving" conditions. In this case, by definition, the momentum flux would be of the order of $L_{\mathrm{AGN}} / c$, consistently to what we infer for SDSS J1148+5251.

Costa et al. (2014b) also show that, when an AGN-driven outflow develops, regardless of its momentum- or energyconserving nature, its morphology is highly anisotropic and it strongly depends on the cosmological environment. In particular, the outflow develops preferably along "lower density" paths, free from filamentary cosmic inflows or from regions characterised by large amounts of dense gas in the halo or in the host galaxy. Interestingly, by comparing the maps in Fig. 4 and the top panel of Fig. 10, we note that the peak of the outflow component does not coincide with the peak of the narrow extended component. On the contrary, the narrow extended component (top panel of Fig. 10) peaks to the south-east with respect to the quasar position, where the outflow appears suppressed. If assuming that the emission within $v \in(-200,200) \mathrm{km} \mathrm{s}^{-1}$ is not significantly contaminated by the outflow, this result would support the scenario in which the outflow develops preferably in directions avoiding high density regions in the galaxy or in the halo.

We now consider the second class of models of AGN-driven outflows, i.e. models of outflows generated by coupling of the AGN radiation to dusty interstellar medium at galactic $(\sim \mathrm{kpc})$ scales. More specifically, we refer to the work by Thompson et al. (2014), who further developed the physics on which these models are based, demonstrating that dusty radiation-pressure driven shells can reach high velocities $\left(v_{\text {out }} \gg v_{\text {esc }}\right)$. The predictions of Thompson et al. (2014) are qualitatively consistent with our observations of the outflow in SDSS J1148+5251, as shown by a comparison between our Fig. 8 and Fig. 3 of their paper. In particular, the decreasing trend with $R$ and the modest (although lower limit) value of the momentum flux inferred for SDSS J1148+5251 could be explained by dusty radiationpressure driven shells without resorting to extreme ISM conditions such as $\tau_{\mathrm{IR}} \gg 1$. Our resolved FIR continuum observations (Fig. 12) show that large amounts of dust are indeed present in this source. However, the FIR emission, indicating the presence of warm dust, only extends up to maximum radial distances of $r \simeq 10 \mathrm{kpc}$ from the nucleus, while the outflow extends up to $r \simeq 30 \mathrm{kpc}$.

We finally consider the possibility that a previous episode of radio-jets may have driven the observed outflow. Carilli et al. (2004) imaged the radio continuum of SDSS J1148+5251 at $1.4 \mathrm{GHz}$ with the very large array (VLA), and they found that this source is radio-quiet, with a radio continuum emission consistent with being primarily powered by star formation. Therefore, if radio jets are responsible for the observed

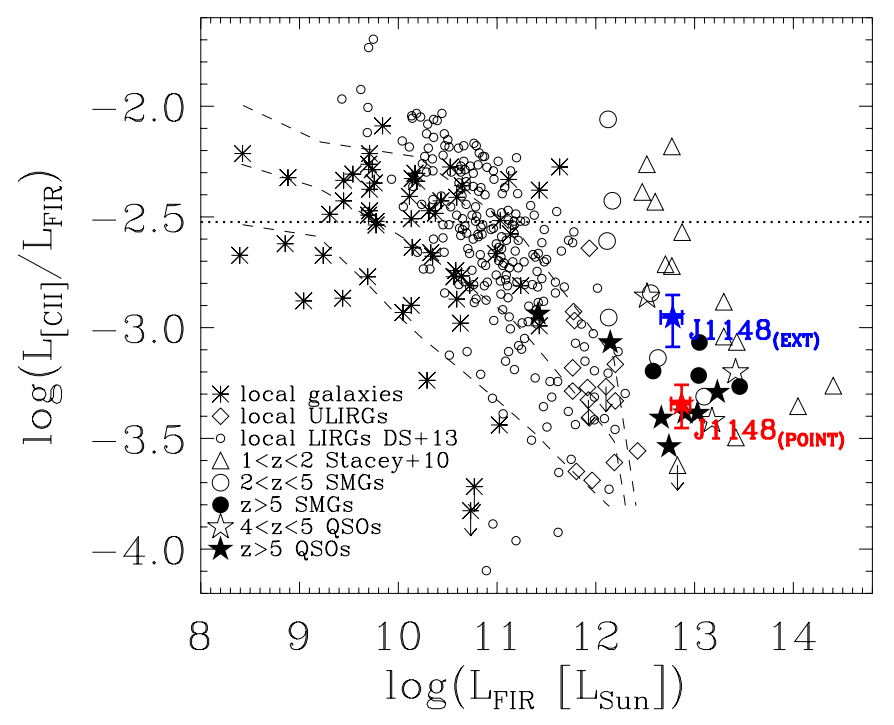

Fig. 13. [C II] /FIR luminosity ratio as a function of FIR luminosity, with our new estimate of $L_{[\mathrm{CII}]} / L_{\mathrm{FIR}}$ in SDSS J1148+5251, obtained separately for the extended and compact components, as explained in Sect. 4.2. For comparison, the plot also includes previous ISO observations of local star-forming galaxies (asterisks, Malhotra et al. 2001) and of local ULIRGs (diamonds, Luhman et al. 2003) along with their median and scatter (dashed lines), recent Herschel/PACS observations of local LIRGs (small open circles, Díaz-Santos et al. 2013), the CSOZEUS survey of star forming and active galaxies at redshift $1<z<2$ (triangles, Stacey et al. 2010), observations of sub-millimetre galaxies (SMGs) at $2<z<5$ (open circles, Ivison et al. 2010; De Breuck et al. 2014; Valtchanov et al. 2011; Cox et al. 2011; Swinbank et al. 2012; Carniani et al. 2013), sub-millimetre galaxies (SMGs) at $z>5$ (filled circles, Riechers et al. 2013, 2014; Neri et al. 2014; Rawle et al. 2014), quasars at $2<z<5$ (open stars, Maiolino et al. 2009; Wagg et al. 2010; Carniani et al. 2013), and $z>5$ quasars (filled stars, Venemans et al. 2012; Wang et al. 2013; Willott et al. 2013). The dotted horizontal line indicates the Milky Way value $\left(L_{[\mathrm{CII}]} / L_{\mathrm{FIR}} \sim 3 \times 10^{-3}\right.$, Carilli \& Walter 2013). All measurements refer to the FIR luminosity as integrated over $42.5-122.5 \mu \mathrm{m}$. Where necessary, we have converted $L_{\mathrm{FIR}(40-500 \mu \mathrm{m})}$ and $L_{\mathrm{TIR}(8-1000 \mu \mathrm{m})}$ into $L_{\mathrm{FIR}(42.5-122.5 \mu \mathrm{m})}$ by using the conversion factors provided by Carilli \& Walter (2013), i.e. $L_{\mathrm{FIR}(40-500 \mu \mathrm{m})} \simeq$ $1.2 L_{\mathrm{FIR}(42.5-122.5 \mu \mathrm{m})}$ and $L_{\mathrm{TIR}(8-1000 \mu \mathrm{m})} \simeq 1.3 L_{\mathrm{FIR}(40-500 \mu \mathrm{m})}$.

large-scale [C II] outflow, they have now disappeared. However, the complex outflow morphology (Fig. 4) and the rather flat trend of velocity as a function of radius (Fig. 5) suggest that a significant contribution from radio jets is unlikely, as radio jets tend to drive very collimated outflows with large velocity gradients across the kpc-scale jets (e.g. Nesvadba et al. 2008).

\subsection{The [C II]-to-FIR luminosity ratio}

In Sects. 3.5 and 3.6 we have shown a method, based on the $u v$ visibility analysis, for decomposing both the narrow $[\mathrm{CII}]$ emission and the FIR continuum emission of SDSS J1148+5251 into an extended (resolved) and a compact (unresolved) component. Following this decomposition, we estimate the [C II]-to-FIR luminosity ratios separately for these two components. We obtain $\log \left(L_{[\mathrm{CII}]} / L_{\mathrm{FIR}}\right)_{\mathrm{EXT}}=-2.95_{-0.13}^{+0.10}$ and $\log \left(L_{[\mathrm{CII}]} / L_{\text {FIR }}\right)_{\text {POINT }}=-3.34_{-0.11}^{+0.09}$. These estimates are compared in Fig. 13 with literature $L_{[\mathrm{CII}]} / L_{\mathrm{FIR}}$ measurements obtained for local and distant galaxies. 
We stress that the $L_{[\mathrm{CII}]} / L_{\mathrm{FIR}}$ values reported in Fig. 13 refer to the compact and extended components of the narrow [C II] emission in SDSS J1148+5251, integrated within (-200, 200) $\mathrm{km} \mathrm{s}^{-1}$. By only selecting the emission within this narrow velocity range, we intend to avoid contamination by the outflow, whose contribution to the [C II] flux may be significant already at velocities $|v|>200 \mathrm{~km} \mathrm{~s}^{-1}$, as shown in Appendix C. However, the reader should be aware that, by selecting a wider velocity range for the [C II] emission, the ratio $L_{\text {[CII] }} / L_{\text {FIR }}$ would naturally increase, especially for the extended component. In particular, by selecting the [C II] emission within $(-300,400) \mathrm{km} \mathrm{s}^{-1}$, we obtain $\log \left(L_{[\mathrm{CII}]} / L_{\mathrm{FIR}}\right)_{\mathrm{EXT}}=-2.79_{-0.14}^{+0.10}$ and $\log \left(L_{[\mathrm{CII}]} / L_{\mathrm{FIR}}\right)_{\text {POINT }}=-3.26_{-0.11}^{+0.09}$, corresponding to an increase of $L_{[\mathrm{CII}]} / L_{\mathrm{FIR}}$ by a factor of $1.5 \pm 0.5$ and by a factor of $1.2 \pm 0.4$ for the extended and compact components, respectively.

The [C II]-to-FIR luminosity ratio is related to the contribution of the [C II] line emission to the cooling of the (neutral) ISM in comparison to the cooling via dust grains as traced by the FIR emission. In local galaxies, early infrared space observatory (ISO) observations and, more recently, Herschel observations, have established a clear trend for decreasing [C II]-to-FIR ratio with increasing FIR luminosity (Luhman et al. 2003; Brauher et al. 2008; Díaz-Santos et al. 2013). The decreasing trend is even more evident for $L_{[\mathrm{CII}]} / L_{\mathrm{FIR}}$ as a function of $L_{\mathrm{FIR}} / M_{\mathrm{H}_{2}}$ (Graciá-Carpio et al. 2011). In particular, in local LIRGs and ULIRGs, $L_{[\mathrm{CII}]} / L_{\mathrm{FIR}}$ drops by about one order of magnitude with respect to normal disk galaxies, and this deficiency is also extended to other FIR fine structure lines such as the [O I] $63 \mu \mathrm{m}$ and [N II] $122 \mu \mathrm{m}$ emission lines.

Lower [C II]-to-FIR ratios are most likely associated with intense star formation activity occurring in compact and warm starbursts (Díaz-Santos et al. 2013). More specifically, in compact starbursts, the main factors that can act to reduce the [C II] emission with respect to the FIR emission are: (i) high gas volume densities $\left(n \gg n_{\mathrm{cr}}^{[\mathrm{CII}]}\right.$ ), for which the level populations of the $\mathrm{C}^{+}$atoms ratio as their statistical weights, and thus the $[\mathrm{C}$ II] intensity becomes almost insensitive to higher gas volume densities and higher FUV fluxes (Wolfire et al. 1989); (ii) high ratios of FUV intensity field to the total hydrogen gas volume density, which would increase the positive charge of dust grains, thereby reducing the efficiency of gas heating via photoelectrons released by dust grains and so the relative efficiency of cooling via [C II] emission with respect to dust cooling (Luhman et al. 2003; Díaz-Santos et al. 2013); (iii) a high ionisation parameter $U$ (which is essentially linked to (ii)) that reduces the opacity of hydrogen atoms (because a high amount of $\mathrm{HI}$ is ionised) with respect to the dust opacity, hence increasing the fraction of UV photons absorbed by dust. Additionally, the incident UV radiation heats the dust to higher temperatures (Abel et al. 2009; Graciá-Carpio et al. 2011). Lower [C II]-to-FIR ratios may also be associated with an increased AGN contribution to the dust heating (and so to $L_{\mathrm{FIR}}$ ) for sources with higher FIR luminosities. According to Sargsyan et al. (2014), the [C II] luminosity is little affected by the presence of an AGN and it traces mostly star formation, while the FIR luminosity can have a significant AGN contribution in addition to the starburst component, resulting therefore in lower $L_{[\mathrm{CII}]} / L_{\mathrm{FIR}}$ for AGN-dominated sources.

However, a relation between the [C II] luminosity and the FIR luminosity has not been clearly established for high redshift infrared luminous galaxies, which display a large scatter in the $L_{[\mathrm{CII}]} / L_{\mathrm{FIR}}$ vs. $L_{\mathrm{FIR}}$ diagram (Graciá-Carpio et al. 2011), in

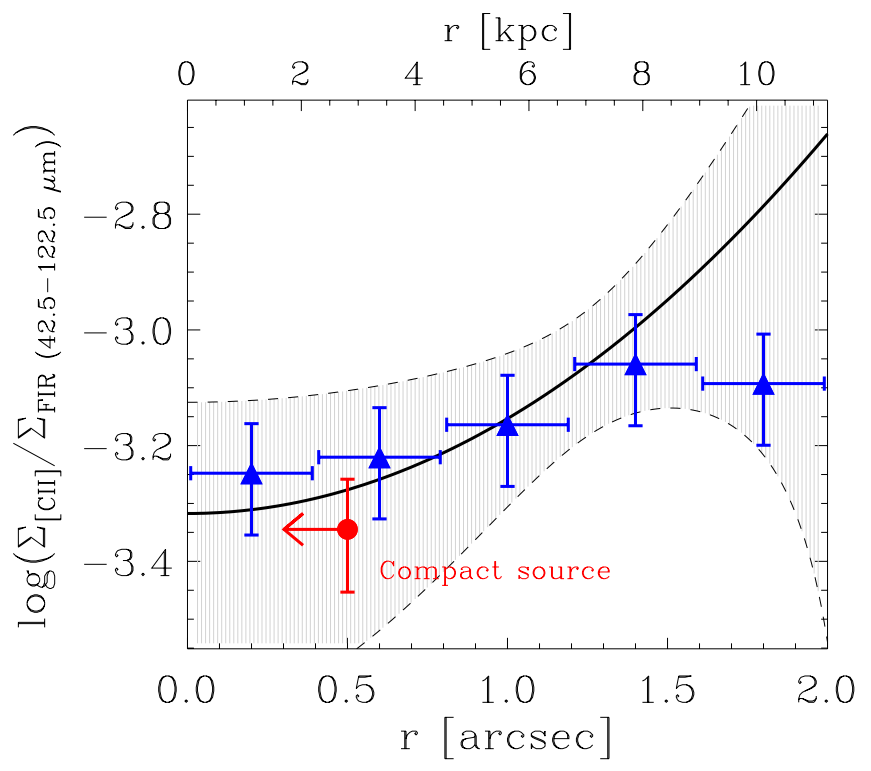

Fig. 14. [C II] /FIR luminosity surface density ratio as a function of the projected distance from the quasar optical position. The black curve represents our model of $\Sigma_{[\mathrm{CII}]} / \Sigma_{\mathrm{FIR}}(r)$ for the extended source, obtained from the analysis of the $u v$ data as explained in Sect. 4.2. The grey shaded region indicates the statistical uncertainty on the $\Sigma_{[\mathrm{CII}]} / \Sigma_{\mathrm{FIR}}$ predicted by the model based on the $u v$ analysis. The blue triangles show the values of $L_{[\mathrm{CII}]} / L_{\mathrm{FIR}}$ calculated at different radii by integrating the extended [C II] and FIR emission over concentric annuli. For comparison, the [C II] /FIR luminosity ratio of the compact (unresolved) component is also shown, plotted at a representative upper limit radius of $F W H M_{\text {beam }} / 2$, where $F W H M_{\text {beam }}$ refers to the beam-size of the FIR continuum observations (Fig. 11).

some cases with values that are completely consistent with local disk galaxies, as also shown by Fig. 13. We find that, in the case of SDSS J1148+5251, the [C II] -to-FIR ratio in the compact source is lower than in the extended component, by a factor of $\sim 2.5$ (Fig. 13). We note that a similar result has been obtained by Díaz-Santos et al. (2014) for the nuclear and extended components of local LIRGs observed with Herschel/PACS. This finding is consistent with a picture in which the nuclear [C II] and FIR emissions in SDSS J1148+5251 are excited by a compact "hyper-starburst" (Walter et al. 2009), similar to the nuclear regions of local ULIRGs, while the extended components trace more diffuse, "disk-like" star formation.

In Fig. 14 we show the [C II] -to-FIR surface density ratio as a function of the projected distance from the quasar. The black curve is obtained by modelling in the $u v$ data the [C II] and FIR extended components with circular Gaussian distributions (Sects. 3.5 and 3.6), and it corresponds to:

$\frac{\Sigma_{[\mathrm{CII}]}}{\Sigma_{\mathrm{FIR}}}(r)=\frac{L_{[\mathrm{CII}], \mathrm{ext}}}{L_{\mathrm{FIR}, \mathrm{ext}}}\left(\frac{\sigma_{\mathrm{FIR}}^{2}}{\sigma_{[\mathrm{CII}]}^{2}}\right) \exp \left[-r^{2}\left(\frac{1}{2 \sigma_{[\mathrm{CII}]}^{2}}-\frac{1}{2 \sigma_{\mathrm{FIR}}^{2}}\right)\right]$,

where $L_{[\mathrm{CII}] \text {,ext }}=(6.7 \pm 0.7) \times 10^{9} L_{\odot}, L_{\mathrm{FIR}, \mathrm{ext}}=(6.0 \pm 1.5) \times$ $10^{12} L_{\odot}, \sigma_{[\mathrm{CII}]}=(1.32 \pm 0.13)$ arcsec and $\sigma_{\mathrm{FIR}}=(0.9 \pm 0.2)$ arcsec, as derived from the $u v$ analysis. We remind that this model of $\Sigma_{[\mathrm{CII}]} / \Sigma_{\mathrm{FIR}}(r)$ is based on the narrow [C II] emission integrated within $(-200,200) \mathrm{km} \mathrm{s}^{-1}$, because in this velocity range the [C II] flux contamination by the outflow is low $(\$ 20 \%$, according to the spectral decomposition shown in Fig. 1). Considering a larger velocity range for the [C II] emission would have as a main effect an upward shift of the relationship in Fig. 14. In particular, if taking into account the $[\mathrm{C} \mathrm{II}]$ emission within 
$(-300,400) \mathrm{km} \mathrm{s}^{-1}, \Sigma_{[\mathrm{CII}]} / \Sigma_{\mathrm{FIR}}$ increases, at every radius, by a factor of $\sim 1.4$.

The blue triangles in Fig. 14 show the $\Sigma_{[\mathrm{CII}]} / \Sigma_{\mathrm{FIR}}(r)$ ratio obtained by integrating the $[\mathrm{C} \mathrm{II}]$ extended emission in the top panel of Fig. 10 over concentric annuli with $\Delta r=0.4$ arcsec. These values follow quite well the black curve, at least up to $r \simeq 8 \mathrm{kpc}$. We also show in Fig. 14 the $L_{[\mathrm{CII}]} / L_{\mathrm{FIR}}$ ratio obtained for the compact source, plotted in correspondence of an upper limit-radius of $0.5 \operatorname{arcsec}(2.8 \mathrm{kpc})$, corresponding to half beam size. Interestingly, the [C II]-to-FIR ratio measured for the compact source is consistent within the one-sigma uncertainty with the value obtained for the extended source at a comparable $r$, suggesting that the two components may be powered by a similar mechanism (i.e. intense and compact star formation), at least up to $\sim 5 \mathrm{kpc}$ distance from the nucleus. At larger $r$, i.e. $5<r[\mathrm{kpc}]<10, \Sigma_{[\mathrm{CII}]} / \Sigma_{\mathrm{FIR}}(r)$ increases to values more compatible with the disks of local galaxies (although still lower than the values measured in the Milky Way and in the bulk of local galaxies, see Fig. 13), hinting at a less extreme star formation activity occurring on these scales.

The discovery of extended star formation (at least up to scales of $r \sim 10 \mathrm{kpc}$ ) in SDSS J1148+5251 challenges the scenario presented by previous observations of high redshift quasar host galaxies, which suggested that in this class of sources both the star formation and the cold gas reservoirs are confined within less than a few kiloparsecs from the nucleus (Carilli \& Walter 2013). Quite interestingly, the possibility of a diffuse and extended mode of star formation in high- $z$ massive galaxies was predicted by Hatch et al. (2008), who discovered a large (60 kpc) halo of diffuse ultraviolet light, powered by a young stellar population, surrounding the Spiderweb Galaxy, a radio galaxy at $z=$ 2. Understanding the origin of the [C II] emission at $r \gtrsim 10 \mathrm{kpc}$ and its connection with star formation is however more complex, and possible explanations are suggested in Sect. 4.3.

\subsection{Origin of the [C II] emission at $r \gtrsim 10 \mathrm{kpc}$}

Figure 14 shows that our model uncertainty for the [C II] -toFIR surface density ratio increases significantly for $r \gtrsim 10 \mathrm{kpc}$, and, in particular, no constraints can be put on $\Sigma_{[\mathrm{CII}]} / \Sigma_{\mathrm{FIR}}$ at such large radii. This is due to the fact that, above $r \sim 8-10 \mathrm{kpc}$, the FIR continuum drops below our sensitivity and only [C II] is detected, as it can be clearly seen by comparing the upper panels of Figs. 10 and 12. Previous studies have suggested that [C II] emission is a good tracer of star formation in galaxies regardless of their classification as starbursts, AGN hosts or composite galaxies (e.g. Sargsyan et al. 2014; De Looze et al. 2014). However, the abrupt drop of FIR surface density that we observe in SDSS J1148+5251 at $r \gtrsim 10 \mathrm{kpc}$ may indicate that the [C II] flux in this source and on such large scales does not correlate with star formation (and so with the FIR emission) in the same way as in the nuclear regions. In other words, extended [C II] emission at $r \gtrsim 10 \mathrm{kpc}$ may trace a different regime of star formation with respect to the central regions of the galaxy $(r<5 \mathrm{kpc})$ and also with respect to the regions at $5<r[\mathrm{kpc}]<10$.

Furthermore, at $r \gtrsim 10 \mathrm{kpc}$, a significant amount of [C II] may trace a different ISM phase than dense PDRs. For example, [C II] may arise from diffuse HI (mostly cold neutral medium, CNM), diffuse molecular clouds and, in minor measure, diffuse warm ionised medium (WIM). This behaviour of [C II] has been observed in low metallicity systems such as Haro 11 and in the outskirts of the Milky Way and other local galaxies (Kramer et al. 2013; Parkin et al. 2013; Pineda et al. 2013; Langer et al. 2014). In particular, Kramer et al. (2013) have shown that the $\mathrm{CNM}$ contribution to $[\mathrm{C} \mathrm{II}]$ in the outer regions of M33 increases by $\sim 25 \%$ with respect to the inner region (i.e. $r<2 \mathrm{kpc}$ ). This effect is accompanied by an increase of $L_{[\mathrm{CII}]} / L_{\mathrm{FIR}}$ by almost a factor of 4 for $r>4.5 \mathrm{kpc}$, similarly to what we observe in SDSS J1148+5251, although in M33 the transition occurs at much smaller scales. Moreover, Pineda et al. (2013) and Langer et al. (2014) have shown that, in our Galaxy, a significant amount of [C II] stems from CNM ( 20\%) and from "CO-dark" molecular gas $(\sim 30 \%)$, which dominates the gas mass of diffuse and "warm" molecular clouds (i.e. a transition phase between diffuse $\mathrm{HI}$ clouds and dense CO-bright molecular clouds), and these fractions increase with increasing galactocentric distance. In addition, Vallini et al. (2013) have shown through radiative transfer simulations of the ISM in Himiko (Ouchi et al. 2013) that [C II] emission may arise from the CNM residing at the periphery $(r \geq 5 \mathrm{kpc})$ of the galaxy.

However, we note that the [C II] surface density that we measure in the extended component of SDSS J1148+5251 is still three orders of magnitude higher than what observed in the outskirts of local galaxies. This implies that the contribution of different ISM phases to the [C II] emission discussed above is not sufficient for justifying the extended [C II] flux - with no significant associated FIR emission - observed at $r \gtrsim 10 \mathrm{kpc}$ in SDSS J1148+5251. Therefore, although a comparison with the local Universe may help understanding what we observe in SDSS J1148+5251, we should keep in mind that the galactic environment of a luminous quasar at $z>6$ can be very different even from the most extreme galaxies that we observe locally, and even more so from the Milky Way or other local disks such as M33 and M51.

In order to clarify the origin of the extended [C II] emission at $r>10 \mathrm{kpc}$, high sensitivity and multi-wavelength observations are needed. In particular, while our IRAM PdBI observations prove, for the first time, that cold gas (both in outflow and not) extends up to $r \sim 30 \mathrm{kpc}$ in the host galaxy of SDSS J1148+5251, corresponding to about $1 / 3$ of the virial radius estimated for its massive halo (Valiante et al. 2011, 2014), it is still not clear what is the primary mechanism exciting [C II] up to such large distances from the nucleus. In summary, whether associated with dense PDRs or with a more diffuse ISM phase, the extended - and relatively high surface density - [C II ] emission discovered in SDSS $\mathrm{J} 1148+5251$ requires an excitation source. This may be provided by in situ star formation, by the nuclear starburst, by the quasar and/or also by strong shocks (e.g. see Appleton et al. 2013).

\section{Summary and conclusions}

We have presented follow-up C and D configuration IRAM PdBI observations of the [C II] $158 \mu \mathrm{m}$ fine structure line and FIR continuum in the host galaxy of the luminous quasar SDSS J1148+5251 at redshift $z=6.4189$. The total [C II] velocity-integrated flux of $34 \pm 9 \mathrm{Jy} \mathrm{km} \mathrm{s}^{-1}$, estimated by using a spectrum extracted from a circular aperture with a diameter of 8 arcsec, is significantly larger than any previous measurement of the same source. This clearly indicates the presence, in the quasar host galaxy, of additional baryonic mass (in cold gas) that was missed by previous observations with a lower sensitivity to large scale structures. The [C II] spectrum shows broad emission extending from $-1400 \mathrm{~km} \mathrm{~s}^{-1}$ to $1200 \mathrm{~km} \mathrm{~s}^{-1}$ from the systemic velocity, confirming the detection of a powerful outflow by Maiolino et al. (2012). The total continuum-subtracted map 
shows a very extended [C II] source, with filamentary structures extending up to the extreme distance of $r \sim 30 \mathrm{kpc}$ from the position of the quasar.

We have further investigated the properties of the outflow, and we have found that:

- The outflow is resolved at high significance by our observations, revealing an extremely complex morphology, butterfly-like, with filaments extending up to $r \simeq 30 \mathrm{kpc}$ from the nucleus.

- Although the median outflow dynamical time scale is $\tau_{\text {dyn }}^{\text {median }}=25 \mathrm{Myr}$, the distribution of $\tau_{\text {dyn }}$ within the outflow is quite broad, ranging from $\sim 4 \mathrm{Myr}$ to $\sim 100 \mathrm{Myr}$, indicating that the outflow has been in place for at least $100 \mathrm{Myr}$, which is a non-negligible fraction of the age of the Universe at this redshift.

- Our new estimates of the lower limits on the integrated massloss rate, momentum rate and kinetic power of the outflow are of $1400 \pm 300 M_{\odot} \mathrm{yr}^{-1},(1.00 \pm 0.14) L_{\mathrm{AGN}} / c$ and $(1.6 \pm$ $0.2) \times 10^{-3} L_{\mathrm{AGN}}$, respectively.

- We have investigated, for the first time in an external galaxy, the outflow properties as a function of the dynamical time scale and of the projected distance from the quasar. The results suggest that the ejection of gas in this source has not occurred at a constant rate, but most likely through multiple outflow events. In particular, we have found that the bulk of the mass, energy and momentum in this source have been released within the past $\sim 20 \mathrm{Myr}$.

We have discussed possible driving sources for the observed [C II] outflow, finding that quasar feedback is likely the dominant mechanism driving the outflow in SDSS J1148+5251 (although the contribution from extended star formation may be significant). We have discussed the outflow properties in the light of theoretical models for AGN-driven outflows. We have noted that the integrated outflow momentum rate of $(1.00 \pm 0.14) L_{\mathrm{AGN}} / c$ and kinetic power of $(1.6 \pm 0.2) \times 10^{-3} L_{\mathrm{AGN}}$ are quite modest compared to local ULIRGs /quasars and in principle consistent with any of the proposed models of AGN-driven outflows. By considering the mass-loss outflow rate, momentum rate and $\mathrm{ki}$ netic power of the outflow as a function of the distance from the quasar, we have found that observations are qualitatively consistent with radiation-pressure driven dusty shells. However, we have also found that the morphology and distribution of the outflow is consistent with recent cosmological simulations involving energy-driven outflows. Yet, a more quantitative comparison with the latter studies requires simulations matching the properties of this specific object (halo mass, black hole mass).

Quite surprisingly, we have discovered that not only the outflow, but also the quiescent gas traced by [C II] at systemic velocity is extremely extended in this source. More specifically, we have found that the $[\mathrm{C} \mathrm{II}]$ emission within $v \in(-200,200) \mathrm{km} \mathrm{s}^{-1}$ traces two components:

(i) A compact source, not resolved at our spatial resolution of $\sim 1.2$ arcsec, which corresponds to the $1.5 \mathrm{kpc}$ size nuclear "hyper-starburs" detected by previous observations;

(ii) An extended source with a FWHM size of $3.1 \pm 0.3$ arcsec, corresponding to $17.4 \pm 1.7 \mathrm{kpc}$. The map of this component reveals a complex structure, with a prominent filament extending up to $r \simeq 28 \mathrm{kpc}$ to the west (with respect to the quasar position). Strikingly, this extended component contributes to almost $\sim 70 \%$ of the [C II] flux within $v \in$ $(-200,200) \mathrm{km} \mathrm{s}^{-1}$.
By using ancillary deep FIR continuum observations at $\lambda_{\text {rest }}=$ $155 \mu \mathrm{m}$, we have found that also the FIR emission from dust can be disentangled into a compact and a more extended component contributing to $45 \%$ of the total FIR emission at this wavelength. The extended FIR component has a FWHM size of $11 \pm 3 \mathrm{kpc}$, with a tail extending by $r \sim 10 \mathrm{kpc}$ to the west, and it has therefore a smaller extent than the extended [C II] source.

We have estimated the [C II] -to-FIR surface density ratio as a function of the distance from the quasar, and we have found that, within $r \lesssim 5 \mathrm{kpc}, \Sigma_{[\mathrm{CII}]} / \Sigma_{\mathrm{FIR}}$ is low $\left(\simeq 6 \times 10^{-4}\right)$ and not significantly different between the compact and the extended components, suggesting that both components may be powered by an intense and compact starburst. At intermediate radii, i.e. $5<r[\mathrm{kpc}]<10, \Sigma_{[\mathrm{CII}]} / \Sigma_{\mathrm{FIR}}$ increases up to $\simeq 1.0 \times 10^{-3}$, supporting the hypothesis of less extreme star formation activity on these scales. At larger radii, i.e. $r \gtrsim 10 \mathrm{kpc}$, the FIR continuum drops below our sensitivity and only [C II] is detected. This indicates that at such large distances from the galaxy centre, the [C II] emission does not correlate with FIR continuum and with star formation in the same way as in the central regions. Moreover, similarly to the outskirts of local galaxies and to low metallicity systems, a large fraction of [C II] at $r \gtrsim 10 \mathrm{kpc}$ may arise from different phases of the ISM than dense PDRs, such as diffuse atomic or molecular gas clouds. However, how [C II] emission can be excited up to such large distances from the quasar and from the intense central starburst remains obscure, and additional observations with the future facilities such as the northern extended millimetre array (NOEMA) are needed for answering this question.

Acknowledgements. We thank the referee, Carlos De Breuck, for providing constructive comments that have significantly helped to improve the paper. We also thank Rosa Valiante and Christian Leipski for useful discussion. C.C. acknowledges support by the Isaac Newton Studentship and by STFC funding. This work is based on observations carried out with the IRAM Plateau de Bure Interferometer. IRAM is supported by INSU/CNRS (France), MPG (Germany) and IGN (Spain). In this paper we use the IDL SED fitting code developed by Caitlin M. Casey (Casey 2012).

\section{References}

Aalto, S., Garcia-Burillo, S., Muller, S., et al. 2012, A\&A, 537, A44 Abel, N. P., Dudley, C., Fischer, J., Satyapal, S., \& van Hoof, P. A. M. 2009, ApJ, 701, 1147

Alatalo, K., Blitz, L., Young, L. M., et al. 2011, ApJ, 735, 88

Appleton, P. N., Guillard, P., \& Boulanger, F. E. A. 2013, ApJ, 777, 66

Bertoldi, F., Carilli, C. L., Cox, P., et al. 2003, A\&A, 406, L55

Brauher, J. R., Dale, D. A., \& Helou, G. 2008, ApJS, 178, 280

Calura, F., Gilli, R., Vignali, C., et al. 2014, MNRAS, 438, 2765

Carilli, C. L., \& Walter, F. 2013, ARA\&A, 51, 105

Carilli, C. L., Walter, F., Bertoldi, F., et al. 2004, AJ, 128, 997

Carniani, S., Marconi, A., Biggs, A., et al. 2013, A\&A, 559, A29

Casey, C. M. 2012, MNRAS, 425, 3094

Castro-Carrizo, A., \& Neri, R. 2010, IRAM Plateau de Bure Interferometer Data Reduction Cookbook

Cicone, C., Feruglio, C., Maiolino, R., et al. 2012, A\&A, 543, A99

Cicone, C., Maiolino, R., Sturm, E., et al. 2014, A\&A, 562, A21

Combes, F., García-Burillo, S., \& Casasola, V. E. A. 2013, A\&A, 558, A124

Contursi, A., Poglitsch, A., Grácia Carpio, J., et al. 2013, A\&A, 549, A118

Costa, T., Sijacki, D., \& Haehnelt, M. 2014a, MNRAS, submitted [arXiv: 1411.0678]

Costa, T., Sijacki, D., \& Haehnelt, M. G. 2014b, MNRAS, 444, 2355

Costa, T., Sijacki, D., Trenti, M., \& Haehnelt, M. G. 2014c, MNRAS, 439, 2146

Cox, P., Krips, M., Neri, R., et al. 2011, ApJ, 740, 63

De Breuck, C., Williams, R. J., Swinbank, M., et al. 2014, A\&A, 565, A59

De Looze, I., Cormier, D., Lebouteiller, V., et al. 2014, A\&A, 568, A62

Decarli, R., Walter, F., Yang, Y., et al. 2012, ApJ, 756, 150

Díaz-Santos, T., Armus, L., Charmandaris, V., et al. 2013, ApJ, 774, 68

Díaz-Santos, T., Armus, L., \& Charmandaris, V. E. A. 2014, ApJ, 788, L17

Draine, B. T. 2006, ApJ, 636, 1114

Emonts, B. H. C., Norris, R. P., \& Feain, I. E. A. 2011, MNRAS, 415, 655 
Emonts, B. H. C., Norris, R. P., Feain, I., et al. 2014, MNRAS, 438, 2898 Fabian, A. C. 2012, ARA\&A, 50, 455

Fan, X., Strauss, M. A., Schneider, D. P., et al. 2003, AJ, 125, 1649 Faucher-Giguère, C.-A., \& Quataert, E. 2012, MNRAS, 425, 605 Feruglio, C., Maiolino, R., Piconcelli, E., et al. 2010, A\&A, 518, L155 Feruglio, C., Fiore, F., Maiolino, R., et al. 2013a, A\&A, 549, A51 Feruglio, C., Fiore, F., \& Piconcelli, E. E. A. 2013b, A\&A, 558, A87 Fischer, J., Sturm, E., González-Alfonso, E., et al. 2010, A\&A, 518, L41 Gallerani, S., Maiolino, R., Juarez, Y., \& Nagao, T. E. A. 2010, A\&A, 523, A85 Gallerani, S., Neri, R., \& Maiolino, R. E. A. 2012, A\&A, 543, A114 Gallerani, S., Ferrara, A., Neri, R., \& Maiolino, R. 2014, MNRAS, 445, 2848 García-Burillo, S., Combes, F., \& Usero, A., et al. 2014, A\&A, 567, A125 Graciá-Carpio, J., Sturm, E., Hailey-Dunsheath, S., et al. 2011, ApJ, 728, L7 Hailey-Dunsheath, S., Nikola, T., Stacey, G. J., et al. 2010, ApJ, 714, L162 Hatch, N. A., Overzier, R. A., Röttgering, H. J. A., Kurk, J. D., \& Miley, G. K. 2008, MNRAS, 383, 931

Hopkins, P. F. \& Elvis, M. 2010, MNRAS, 401, 7

Ivison, R. J., Morrison, G. E., Biggs, A. D., et al. 2008, MNRAS, 390, 1117

Ivison, R. J., Swinbank, A. M., Swinyard, B., et al. 2010, A\&A, 518, L35

Ivison, R. J., Smail, I., Amblard, A., et al. 2012, MNRAS, 425, 1320

Kennicutt, Jr., R. C. 1998, ARA\&A 36, 189

King, A. 2003, ApJ, 596, L27

King, A. 2005, ApJ, 635, L121

King, A. R. 2010, MNRAS, 402, 1516

Kramer, C., Abreu-Vicente, J., García-Burillo, S., et al. 2013, A\&A, 553, A114

Kreckel, K., Armus, L., Groves, B., et al. 2014, ApJ, 790, 26

Langer, W. D., Velusamy, T., Pineda, J. L., et al. 2010, A\&A, 521, L17

Langer, W. D., Velusamy, T., Pineda, J. L., Willacy, K., \& Goldsmith, P. F. 2014, A\&A, 561, A122

Lapi, A., Cavaliere, A., \& Menci, N. 2005, ApJ, 619, 60

Leipski, C., Meisenheimer, K., Klaas, U., et al. 2010, A\&A, 518, L34

Leipski, C., Meisenheimer, K., \& Walter, F., et al. 2013, ApJ, 772, 103

Leipski, C., Meisenheimer, K., Walter, F., \& Klaas, U. E. A. 2014, ApJ, 785, 154

Luhman, M. L., Satyapal, S., Fischer, J., et al. 2003, ApJ, 594, 758

Maiolino, R., Cox, P., Caselli, P., et al. 2005, A\&A, 440, L51

Maiolino, R., Caselli, P., Nagao, T., et al. 2009, A\&A, 500, L1

Maiolino, R., Gallerani, S., Neri, R., et al. 2012, MNRAS, 425, L66

Malhotra, S., Kaufman, M. J., Hollenbach, D., et al. 2001, ApJ, 561, 766

Menci, N., Fontana, A., Giallongo, E., Grazian, A., \& Salimbeni, S. 2006, ApJ, 647,753

Menci, N., Fiore, F., Puccetti, S., \& Cavaliere, A. 2008, ApJ, 686, 219

Neri, R., Downes, D., Cox, P., \& Walter, F. 2014, A\&A, 562, A35

Nesvadba, N. P. H., Lehnert, M. D., De Breuck, C., Gilbert, A. M., \& van Breugel, W. 2008, A\&A, 491, 407

Nesvadba, N. P. H., Neri, R., De Breuck, C., et al. 2009, MNRAS, 395, L16

Ouchi, M., Ellis, R., Ono, Y., \& Nakanishi, K. E. A. 2013, ApJ, 778, 102
Parkin, T. J., Wilson, C. D., Schirm, M. R. P., \& Baes, M. E. A. 2013, ApJ, 776, 65

Pineda, J. L., Langer, W. D., Velusamy, T., \& Goldsmith, P. F. 2013, A\&A, 554, A103

Planck Collaboration XVI. 2014, A\&A, 571, A16

Rawle, T. D., Egami, E., \& Bussmann, R. S., et al. 2014, ApJ, 783, 59

Riechers, D. A., Walter, F., Bertoldi, F., et al. 2009, ApJ, 703, 1338

Riechers, D. A., Bradford, C. M., Clements, D. L., et al. 2013, Nature, 496, 329

Riechers, D. A., Carilli, C. L., Capak, P. L., et al. 2014, ApJ, accepted [arXiv: 1404.7159]

Roth, N., Kasen, D., Hopkins, P. F., \& Quataert, E. 2012, ApJ, 759, 36

Sargsyan, L., Samsonyan, A., Lebouteiller, V., et al. 2014, ApJ, 790, 15

Schneider, R., Bianchi, S., Valiante, R., Risaliti, G., \& Salvadori, S. 2014 [arXiv: 1402.2279]

Spoon, H. W. W., Farrah, D., \& Lebouteiller, V., et al. 2013, ApJ, 775, 127

Stacey, G. J., Hailey-Dunsheath, S., Ferkinhoff, C., et al. 2010, ApJ, 724, 957

Sturm, E., González-Alfonso, E., Veilleux, S., et al. 2011, ApJ, 733, L16

Swinbank, A. M., Karim, A., Smail, I., et al. 2012, MNRAS, 427, 1066

Thompson, T. A., Fabian, A. C., Quataert, E., \& Murray, N. 2014 [arXiv: 1406.5206]

Valiante, R., Schneider, R., Salvadori, S., \& Bianchi, S. 2011, MNRAS, 416, 1916

Valiante, R., Schneider, R., Maiolino, R., Salvadori, S., \& Bianchi, S. 2012, MNRAS, 427, L60

Valiante, R., Schneider, R., Salvadori, S., \& Gallerani, S. 2014, MNRAS, 444, 2442

Vallini, L., Gallerani, S., Ferrara, A., \& Baek, S. 2013, MNRAS, 433, 1567

Valtchanov, I., Virdee, J., Ivison, R. J., et al. 2011, MNRAS, 415, 3473

Veilleux, S., Cecil, G., \& Bland-Hawthorn, J. 2005, ARA\&A, 43, 769

Veilleux, S., Meléndez, M., Sturm, E., et al. 2013, ApJ, 776, 27

Velusamy, T., \& Langer, W. D. 2014 [arXiv: 1409. 4788]

Velusamy, T., Langer, W. D., \& Pineda, J. L., et al. 2010, A\&A, 521, L18

Venemans, B. P., McMahon, R. G., Walter, F., et al. 2012, ApJ, 751, L25

Villar-Martín, M., Vernet, J., di Serego Alighieri, S., et al. 2003, MNRAS, 346, 273

Wagg, J., Carilli, C. L., Wilner, D. J., et al. 2010, A\&A, 519, L1

Walter, F., Bertoldi, F., \& Carilli, C., et al. 2003, Nature, 424, 406

Walter, F., Carilli, C., Bertoldi, F., et al. 2004, ApJ, 615, L17

Walter, F., Riechers, D., Cox, P., et al. 2009, Nature, 457, 699

Wang, R., Wagg, J., Carilli, C. L., et al. 2013, ApJ, 773, 44

Willott, C. J., McLure, R. J., \& Jarvis, M. J. 2003, ApJ, 587, L15

Willott, C. J., Omont, A., \& Bergeron, J. 2013, ApJ, 770, 13

Wolfire, M. G., Hollenbach, D., \& Tielens, A. G. G. M. 1989, ApJ, 344, 770

Wolfire, M. G., Hollenbach, D., \& McKee, C. F. 2010, ApJ, 716, 1191

Zubovas, K., \& King, A. 2012, ApJ, 745, L34

Zubovas, K., \& King, A. R. 2014, MNRAS, 439, 400

Pages 19 to 21 are available in the electronic edition of the journal at http://www . aanda. org 


\section{Appendix A: Additional FIR continuum source}

Herschel observations revealed the presence of an additional FIR continuum source, north-west of SDSS J1148+5251, at a distance of $\sim 10 "$ (Leipski et al. 2010, 2013, 2014). This northwestern source is detected at the $\sim 5 \sigma$ level in our PdBI observations. More specifically, Fig. A.1 shows that a FIR continuum source at a distance of 10.5 " from SDSS J1148+5251 is independently detected in both our FIR continuum maps at $256 \mathrm{GHz}$ $(4.5 \sigma)$ and at $261 \mathrm{GHz}(5.6 \sigma)$.

In Sect. 3.1 we have evidenced a $2 \sigma$ inconsistency between our new FIR continuum flux density estimate at $256 \mathrm{GHz}$ of $3.3 \pm 0.7 \mathrm{mJy}$ and the flux density of $4.8 \pm 0.6 \mathrm{mJy}$ expected at the same observed frequency from the MAMBO-II bolometric observations by Bertoldi et al. (2003). Such small discrepancy can be fully explained by the presence of the north-western source contaminating the MAMBO-II flux measurement. We further test this hypothesis by performing a fitting of the FIR SED of SDSS J1148+5251, in which we select only the continuum measurements available in the literature that are not contaminated by the north-western source or that have been corrected for this effect (Fig. A.2). In particular, we select the $100 \mu \mathrm{m}$ and $160 \mu \mathrm{m}$ Herschel/PACS observations by Leipski et al. (2013) (corrected for the contaminating source), the $1.1 \mathrm{~mm}$ PdBI observations by Gallerani et al. (2014) (observed frequency of $262 \mathrm{GHz}$ ), and the $1.3 \mathrm{~mm}$ and $2.8 \mathrm{~mm}$ PdBI observations by Riechers et al. (2009) (observed frequencies of $225 \mathrm{GHz}$ and $109 \mathrm{GHz}$, respectively). We exclude from the SED fitting our PdBI data point at $256 \mathrm{GHz}$ (shown as a red star in Fig. A.2), as the purpose of the fit is showing the consistency of our continuum measurement with the previous observations, once corrected for the contaminating north-western source. The FIR SED in Fig. A.2 is fit with a single temperature modified blackbody combined with a mid infrared (MIR) power law, following Casey (2012). By fixing the emissivity $\beta=1.6$, we obtain a dust temperature of $64 \mathrm{~K}$. We note that our PdBI continuum observation at $256 \mathrm{GHz}$ sits perfectly on the SED model in Fig. A.2.

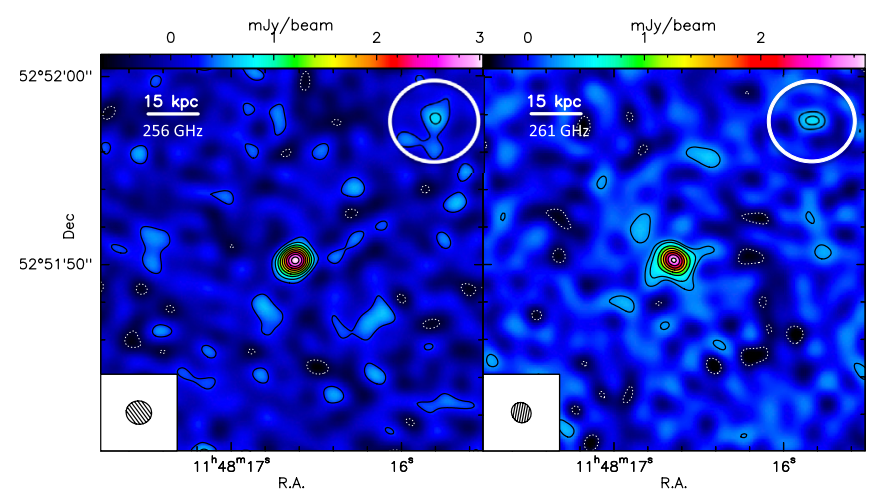

Fig. A.1. IRAM PdBI maps of the FIR continuum showing the marginal detection of an additional source in the field, north-west of SDSS J1148+5251, at a projected distance of 10.5 arcsec, originally discovered by Herschel. Left: the map on the left panel has been obtained by collapsing the line-free channels of the dataset at $256 \mathrm{GHz}$ as explained in Sect. 3.1. The synthesised beam is $1.3^{\prime \prime} \times 1.2^{\prime \prime}$. Negative and positive contours correspond to $-3 \sigma, 2 \sigma, 4 \sigma$ to $18 \sigma$ in steps of $2 \sigma$ $\left(1 \sigma \mathrm{rms}\right.$ noise is $\left.0.159 \mathrm{mJy}^{\text {beam }}{ }^{-1}\right)$. Right: the map on the right panel has been obtained by merging the two ancillary datasets at $262 \mathrm{GHz}$ and $259.4 \mathrm{GHz}$ (average observed frequency of $260.7 \mathrm{GHz}$ ). This is the same map as Fig. 11, which is employed for studying the spatial extent of the FIR continuum. The synthesised beam is $1.1^{\prime \prime} \times 1.0^{\prime \prime}$. Negative and positive contours correspond to $-3 \sigma, 3 \sigma, 5 \sigma$ to $40 \sigma$ in steps of $5 \sigma$ $\left(1 \sigma \mathrm{rms}\right.$ noise is $0.082 \mathrm{mJy}$ beam $\left.^{-1}\right)$.

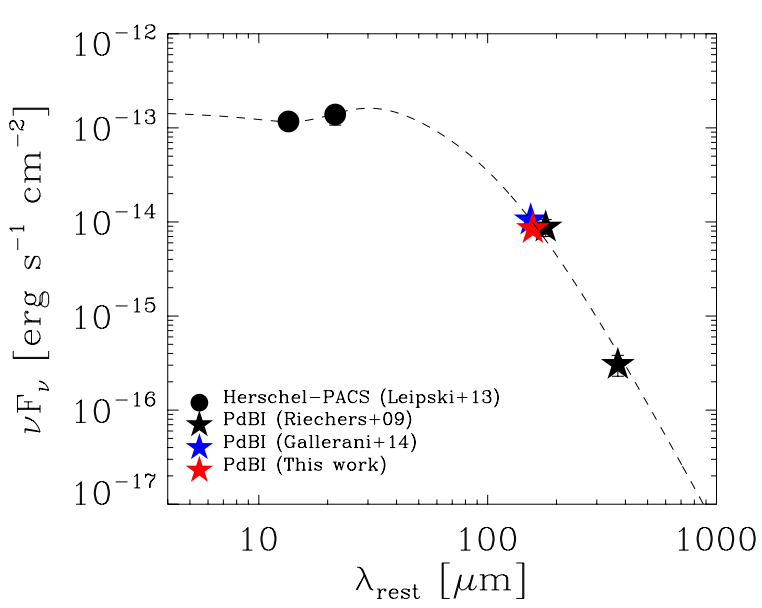

Fig. A.2. FIR SED of SDSS J1148+5251, where only the continuum measurements corrected for the contaminating north-west source are shown, i.e. the Herschel/PACS observations by Leipski et al. (2013), the PdBI observations by Riechers et al. (2009) and Gallerani et al. (2014), and our new PdBI observations at $256 \mathrm{GHz}$ (Sect. 3.1). Different symbols have been used for different datasets (see legend at the bottom-left corner of the plot). The FIR SED has been fit with a single dust temperature modified blackbody with fixed emissivity $\beta=1.6$, combined with a MIR power law, following Casey (2012). Our new PdBI data point at $256 \mathrm{GHz}$ has been excluded from the fit, as explained in the text.

We then use the FIR SED fit model for estimating the expected MAMBO-II flux density, by taking into account the spectral response of the bolometer (transmission curve). We obtain an expected MAMBO-II flux density of $3.8 \mathrm{mJy}$, i.e. significantly lower than the value reported by Bertoldi et al. (2003), which would in turn predict a flux density at $256 \mathrm{GHz}$ of $3.6 \pm 0.4 \mathrm{mJy}$, consistent with our PdBI measurement.

\section{Appendix B: Spectral features in the extended [CII] source}

In Table B.1 we report the results of the spectral fitting to the bright features A-F of the extended [C II] $158 \mu \mathrm{m}$ source, shown in Fig. 3. It is immediately evident from Table B.1 that the features A-F are characterised, on average, by high velocity

Table B.1. Results of the Gaussian fits to the spectra extracted from different positions on the source shown in Fig. 3

\begin{tabular}{lcccc}
\hline \hline Pos $^{\dagger}$ & $\begin{array}{c}v \\
{\left[\mathrm{~km} \mathrm{~s}^{-1}\right]}\end{array}$ & $\begin{array}{c}\sigma_{v} \\
{\left[\mathrm{~km} \mathrm{~s}^{-1}\right]}\end{array}$ & $\begin{array}{c}S_{\text {peak }} \\
{[\mathrm{mJy}]}\end{array}$ & $\begin{array}{c}I_{v} \\
{\left[\mathrm{Jy} \mathrm{km} \mathrm{s}^{-1}\right]}\end{array}$ \\
\hline $\mathrm{A}$ & $-700 \pm 300$ & $800 \pm 300$ & $0.8 \pm 0.2$ & $1.7 \pm 0.8$ \\
& $1100 \pm 140$ & $230 \pm 150$ & $0.8 \pm 0.4$ & $0.5 \pm 0.4$ \\
$\mathrm{~B}$ & $-170 \pm 40$ & $110 \pm 50$ & $2.7 \pm 1.0$ & $0.7 \pm 0.4$ \\
& $-300 \pm 400$ & $800 \pm 400$ & $0.9 \pm 0.5$ & $1.7 \pm 1.3$ \\
$\mathrm{C}$ & $-1120 \pm 110$ & $210 \pm 120$ & $1.5 \pm 0.7$ & $0.8 \pm 0.6$ \\
& $700 \pm 500$ & $700 \pm 400$ & $1.0 \pm 0.5$ & $1.6 \pm 1.2$ \\
& $130 \pm 100$ & $140 \pm 120$ & $1.4 \pm 1.0$ & $0.5 \pm 0.6$ \\
$\mathrm{D}$ & $-900 \pm 400$ & $600 \pm 400$ & $1.1 \pm 0.5$ & $1.5 \pm 1.3$ \\
& $800 \pm 200$ & $500 \pm 200$ & $1.6 \pm 0.5$ & $1.9 \pm 1.1$ \\
$\mathrm{E}$ & $-1430 \pm 90$ & $130 \pm 90$ & $1.1 \pm 0.6$ & $0.4 \pm 0.3$ \\
& $100 \pm 200$ & $500 \pm 200$ & $1.5 \pm 0.3$ & $2.0 \pm 0.9$ \\
& $1200 \pm 200$ & $300 \pm 200$ & $0.7 \pm 0.6$ & $0.5 \pm 0.5$ \\
$\mathrm{~F}$ & $110 \pm 80$ & $220 \pm 80$ & $1.9 \pm 0.5$ & $1.0 \pm 0.5$ \\
& $1100 \pm 90$ & $100 \pm 90$ & $1.0 \pm 0.7$ & $0.3 \pm 0.3$ \\
& $-800 \pm 500$ & $400 \pm 600$ & $0.4 \pm 0.4$ & $0.3 \pm 0.7$ \\
\hline
\end{tabular}

Notes. ${ }^{(\dagger)}$ Each row corresponds to a Gaussian component. We note that in all cases the spectral fit requires multiple Gaussian components. 
dispersions, which can be as high as $\sigma_{v} \sim 800 \mathrm{~km} \mathrm{~s}^{-1}$, suggesting that they are mostly associated with the outflow discovered by Maiolino et al. (2012). This is confirmed by the analysis of the maps of the broad wings (Sect. 3.4). However, we note that, in correspondence of some positions (e.g. B, C, F), there is also a significant contribution from "narrow" emission at the systemic velocity, hinting at the presence of a very extended "quiescent" (i.e. non outflowing) [C II] component, which is investigated in Sect. 3.5.

\section{Appendix C: Outflow channel maps}

In this section we explain the method we employ for estimating the outflow mass-loss rate using the resolved information provided by our IRAM PdBI observations. We produce channel maps of the [C II] $158 \mu \mathrm{m}$ blue- and red-shifted emission at velocities $v \leq-200 \mathrm{~km} \mathrm{~s}^{-1}$ and $v \geq 200 \mathrm{~km} \mathrm{~s}^{-1}$, respectively. The maps are shown in Fig. C.1. The channel widths vary with velocities, because we aim to obtain an approximately constant signalto-noise for the extended emission in each map. For this reason, at lower velocities, i.e. closer to the $[\mathrm{C} \mathrm{II}]$ emission peak, we use narrow channels with $\Delta v=100 \mathrm{~km} \mathrm{~s}^{-1}$, while at higher velocities, tracing the fainter high-velocity component of the outflow, we integrate over wider channels of $\Delta v \sim 200-500 \mathrm{~km} \mathrm{~s}^{-1}$. The centroid positions of the 48 clumps that we ascribe to the outflow are indicated in Fig. C.1. These are selected to be spatially included within the region corresponding to the total [C II] source shown in Fig. 2 and to have in the maps a signal-to-noise ratio $S N R \geq 3$. We note that we have conservatively excluded from the outflow computation the central components of maps $\mathrm{B} 1\left(\langle v\rangle=-250 \mathrm{~km} \mathrm{~s}^{-1}\right)$ and $\mathrm{R} 1\left(\langle v\rangle=250 \mathrm{~km} \mathrm{~s}^{-1}\right)$, because, due to low velocities probed by these two maps, it is difficult to assess to which extent the central extended emission belongs to the outflow. For similar reasons we have not taken into account the central core emission within $\pm 200 \mathrm{~km} \mathrm{~s}^{-1}$, although there may be a significative outflow contribution of $\sim 20 \%$ even at these low velocities, as suggested by the Gaussian fits to the line profile (Fig. 1). However, we have included in the outflow the central blobs visible in maps B2 and R2 (mean velocities of $\pm 350 \mathrm{~km} \mathrm{~s}^{-1}$ ), because they are offset with respect to the quasar position and appear to be co-spatial with the higher velocity emission in maps B3 and R3, which is undoubtedly tracing the outflow.

For each blob (belonging to the outflow) we calculate from the maps its projected distance from the central quasar and, accordingly, obtain a measure of its dynamical time scale. The dynamical time scale is defined as $\tau_{\text {dyn }}=R / v$, where $R$ is the distance travelled by the clump (assumed equal to the projected distance between the centroid of each clump and the optical position of the quasar) and $v$ is the velocity of the gas. The error on $R$ is estimated differently for resolved and unresolved clumps; in particular, for unresolved clumps, $\sigma_{R}$ is the FWHM of the synthesised beam divided by the signal-to-noise ratio. For resolved clumps, instead, we set $\sigma_{R}=\left(r_{\max }^{2}-\left(F W H M_{\text {beam }} / 2\right)^{2}\right)^{1 / 2}$, where $r_{\max }$ is the maximum (projected) radius of the blob. We conservatively adopt for $v$ the average (projected) velocity of each map. We note that the uncertainty on $v$, defined as $\sigma_{v}=\Delta v / 2$, is obviously higher in the higher velocity maps, where we have integrated the emission over larger velocity channels $(\Delta v)$. The distribution of $\tau_{\text {dyn }}$ that we measure within the outflow is shown in Fig. 6.

To estimate the mass of (atomic) gas in outflow, we first measure the $[\mathrm{C}$ II] flux associated with each outflowing clump, by using apertures centred on the positions of the crosses (indicated in Fig. C.1). We then convert the [C II] fluxes (integrated over their corresponding velocity ranges $\Delta v$ ) into [C II] $158 \mu \mathrm{m}$ luminosities, and the [C II] luminosities into (lower limits on the) atomic
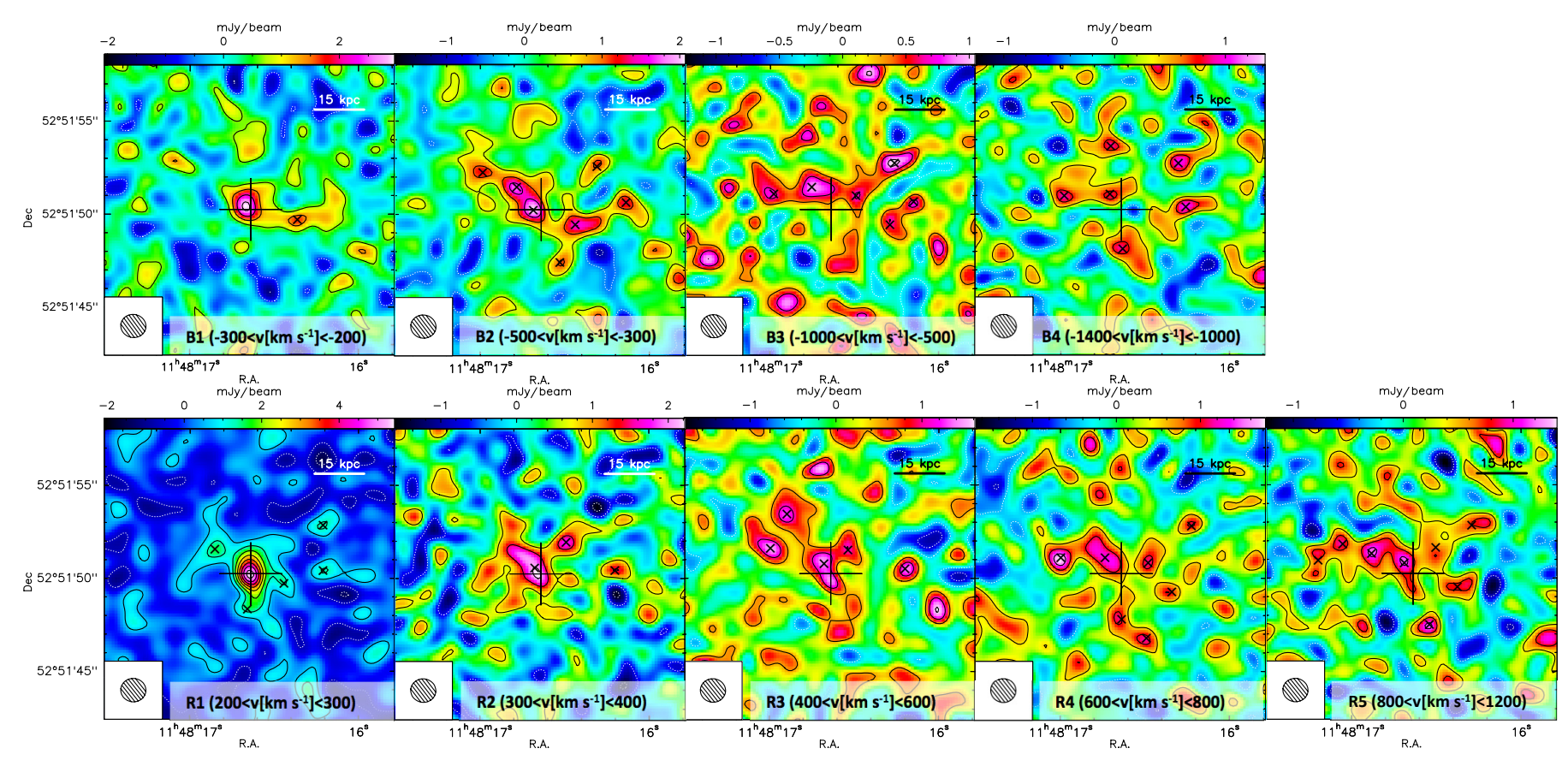

Fig. C.1. IRAM PdBI continuum-subtracted maps of the blue- (top panels) and red- (bottom panels) shifted [C II] $158 \mu \mathrm{m}$ emission of SDSS J1148+5251, obtained at different velocities. The corresponding velocity integration ranges are indicated on each map. Negative and positive contours are in steps of $1.5 \sigma$. In all panels the big central cross indicates the pointing and phase centre, corresponding to the optical position of the quasar. The small crosses mark the position of the 48 blobs that we identified as belonging to the outflow. For each of these clumps in outflow we estimate the dynamical time scale $\tau_{\mathrm{dyn}}=R / v$, where $R$ is the distance from the quasar position, and $v$ is the average velocity of each map. 
gas mass, following Hailey-Dunsheath et al. (2010) as explained in Sect. 3.4. By adding up the mass-loss rate contribution, i.e. $\dot{M}_{\text {out }}=M_{\text {out }} / \tau_{\text {dyn }}$, from all the 48 clumps in outflow, we obtain a total integrated outflow rate of $1400 \pm 300 M_{\odot} \mathrm{yr}^{-1}$. The error is simply $\left(\sum_{i=1}^{i=48} \sigma_{i, \dot{M}_{\text {out }}}^{2}\right)^{1 / 2}$, where $\sigma_{\dot{M}_{\text {out }}}$ is the uncertainty on $\dot{M}_{\text {out }}$ for a given clump in outflow, obtained by propagating errors on $\tau_{\text {dyn }}$ and on the velocity-integrated flux associated with the clump. We note, however, that the uncertainty on the conversion from [C II] luminosity to atomic gas mass is not taken into account in our error estimate.

\section{Appendix D: Moments maps}

We show in Fig. D.1 the first and second moment maps of the [C II] emission within $v \in(-200,200) \mathrm{km} \mathrm{s}^{-1}$, obtained by applying a flux threshold of $2.8 \mathrm{mJy}$. The moments maps suggest that the bulk of $[\mathrm{C}$ II] does not trace gas in a regularly rotating disk. Our IRAM PdBI observations are dominated by the extended [C II] component which, in this velocity range, contributes to $\sim 70 \%$ of the total flux (Sect. 3.5). It is however possible that the compact source, unresolved by our observations, is rotationallysupported, as suggested by Walter et al. (2009). Moreover, the first moment map shows a north-south velocity gradient south of the quasar position, with velocities ranging from $20 \mathrm{~km} \mathrm{~s}^{-1}$ to $100 \mathrm{~km} \mathrm{~s}^{-1}$, whose origin is not clear. In conclusion, no constrains can be put on the total dynamical mass of the system from our [C II] observations.

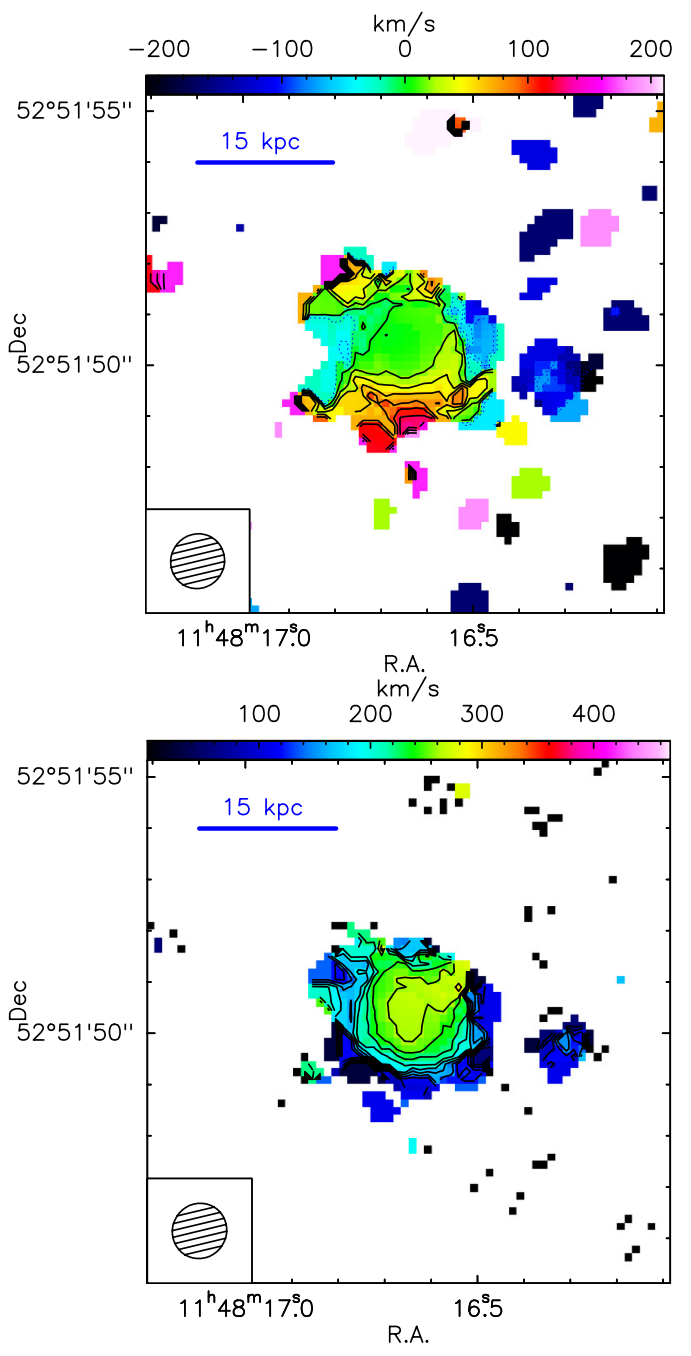

Fig. D.1. First (top) and second (bottom) moment maps obtained within $v \in(-200,200) \mathrm{km} \mathrm{s}^{-1}$ by applying a flux threshold of $2.8 \mathrm{mJy}$. Velocity contours in both maps are in steps of $20 \mathrm{~km} \mathrm{~s}^{-1}$. 